\title{
The transverse momentum dependent distribution functions in the bag model
}

\author{
H. Avakian, ${ }^{1}$ A. V. Efremov, ${ }^{2}$ P. Schweitzer, ${ }^{3}$ and F. Yuan ${ }^{4,5}$ \\ ${ }^{1}$ Thomas Jefferson National Accelerator Facility, Newport News, VA 23606, U.S.A. \\ ${ }^{2}$ Joint Institute for Nuclear Research, Dubna, 141980 Russia \\ ${ }^{3}$ Department of Physics, University of Connecticut, Storrs, CT 06269, U.S.A. \\ ${ }^{4}$ RIKEN BNL Research Center, Building 510A, BNL, Upton, NY 11973, U.S.A. \\ ${ }^{5}$ Nuclear Science Division, Lawrence Berkeley National Laboratory, Berkeley, CA 94720, U.S.A.
}

(Dated: January 2010)

\begin{abstract}
Leading and subleading twist transverse momentum dependent parton distribution functions (TMDs) are studied in a quark model framework provided by the bag model. A complete set of relations among different TMDs is derived, and the question is discussed how model-(in)dependent such relations are. A connection of the pretzelosity distribution and quark orbital angular momentum is derived. Numerical results are presented, and applications for phenomenology discussed. In particular, it is shown that in the valence- $x$ region the bag model supports a Gaussian Ansatz for the transverse momentum dependence of TMDs.
\end{abstract}

PACS numbers: 13.88.+e, 13.85.Ni, 13.60.-r, 13.85.Qk

Keywords: Semi-inclusive deep inelastic scattering, transverse momentum dependent distribution functions

\section{INTRODUCTION}

TMDs are a generalization [1 [4] of parton distribution functions (PDFs) promising to extend our knowledge of the nucleon structure far beyond what we have learned from PDFs about the longitudinal momentum distributions of partons in the nucleon. In addition to the latter, TMDs carry also information on transverse parton momenta and spin-orbit correlations [5 35]. Here longitudinal and transverse refers to the hard momentum flow in the process, for example, in deeply inelastic lepton nucleon scattering (DIS) the momentum of the virtual photon.

TMDs (and/or transverse momentum dependent fragmentation functions) enter the description of leading-twist observables in deeply inelastic reactions [5 7] on which data are available like: semi-inclusive DIS (SIDIS) [36 50], Drell-Yan process [51 53], or hadron production in $e^{+} e^{-}$annihilations [54 57].

The interpretation of these data is not straight-forward though. In SIDIS one deals with convolutions of a priori unknown transverse momentum distributions in nucleon and fragmentation process, and in practice is forced to assume models for transverse parton momenta such as the Gaussian Ansatz [58 67]. In the case of subleading twist observables, one moreover faces the problem that several twist-3 TMDs and fragmentation functions enter the description of one observable [68 77] (we recall that presently factorization is not proven for subleading-twist observables [78]).

In this situation information from models [74 94] is valuable for several reasons. Models can be used for direct estimates of observables, though it is difficult to reliably apply the results, typically obtained at low hadronic scales, to experimentally relevant energies [86]. Another aspect concerns relations among TMDs observed in models [79 83]. Such relations, especially when supported by several models, could be helpful - at least for qualitative interpretations of first data. Furthermore, model results allow to test assumptions made in literature, such as the Gaussian Ansatz for transverse momentum distributions or certain approximations 95 101].

In addition to such practical applications model studies are of interest also because they provide important insights into non-perturbative properties of TMDs. In this context the probably most interesting recent observation in models concerns the pretzelosity distribution function, which in some quark models is related to the difference of the helicity and transversity distributions [80] and, so far, in one model to quark orbital momentum [84] which is, to best of our knowledge, the first 'rigorous' connection of a TMD and quark orbital angular momentum in a model.

The purpose of this work is to study TMDs in the framework of the MIT bag model. We compute in this model all leading- and subleading-twist, time-reversal (T-) even TMDs in Sec. III and address then in Sec. III questions like: how do relations among TMDs arise in a quark model? How many such relations are there in a model? To which extent may one expect such relations to be realized in nature? In Sec. IV we establish a connection of pretzelosity and quark orbital angular momentum in the bag model. In Sec. $\mathrm{V}$ we present and discuss the numerical results, using them, among others, for 'testing' the Gaussian Ansatz or Wandzura-Wilczek-type approximations 98 101. Finally, in Sec. VI we present our conclusions. Some of the results presented here were shown in the proceeding [87].

For convenience and in order to make this work self-contained, in the remainder of this Introduction we include general definitions of TMDs, and introduce relevant notation. 


\section{A. General definitions of TMDs}

Hard processes sensitive to parton transverse momenta like SIDIS are described in terms of light-front correlators

$$
\phi\left(x, \vec{p}_{T}\right)_{i j}=\left.\int \frac{\mathrm{d} z^{-} \mathrm{d}^{2} \vec{z}_{T}}{(2 \pi)^{3}} e^{i p z}\langle N(P, S)| \bar{\psi}_{j}(0) \mathcal{W}(0, z ; \text { path }) \psi_{i}(z)|N(P, S)\rangle\right|_{z^{+}=0, p^{+}=x P^{+}} .
$$

We use light-cone coordinates $a^{ \pm}=\left(a^{0} \pm a^{3}\right) / \sqrt{2}$. In SIDIS the singled-out 3-direction is along the momentum of the hard virtual photon, and transverse vectors like $\vec{p}_{T}$ are perpendicular to it. The path of the symbolically indicated Wilson-link depends on the process [23 25]. In the nucleon rest frame the polarization vector is given by $S=\left(0, \vec{S}_{T}, S_{L}\right)$ with $\vec{S}_{T}^{2}+S_{L}^{2}=1$.

The information content of the correlator (11) is summarized by eight leading-twist TMDs [18], that can be projected out from the correlator (11) as follows (color online: red: T-odd, blue: T-even)

$$
\begin{aligned}
\frac{1}{2} \operatorname{tr}\left[\gamma^{+} \phi\left(x, \vec{p}_{T}\right)\right] & =f_{1}-\frac{\varepsilon^{j k} p_{T}^{j} S_{T}^{k}}{M_{N}} f_{1 T}^{\perp} \\
\frac{1}{2} \operatorname{tr}\left[\gamma^{+} \gamma_{5} \phi\left(x, \vec{p}_{T}\right)\right] & =S_{L} g_{1}+\frac{\vec{p}_{T} \cdot \vec{S}_{T}}{M_{N}} g_{1 T}^{\perp} \\
\frac{1}{2} \operatorname{tr}\left[i \sigma^{j+} \gamma_{5} \phi\left(x, \vec{p}_{T}\right)\right] & =S_{T}^{j} h_{1}+S_{L} \frac{p_{T}^{j}}{M_{N}} h_{1 L}^{\perp}+\frac{\left(p_{T}^{j} p_{T}^{k}-\frac{1}{2} \vec{p}_{T}^{2} \delta^{j k}\right) S_{T}^{k}}{M_{N}^{2}} h_{1 T}^{\perp}+\frac{\varepsilon^{j k} p_{T}^{k}}{M_{N}} h_{1}^{\perp},
\end{aligned}
$$

and by the subleading twist TMDs [29]

$$
\begin{aligned}
\frac{1}{2} \operatorname{tr}\left[1 \phi\left(x, \vec{p}_{T}\right)\right] & =\frac{M_{N}}{P^{+}}\left[e-\frac{\varepsilon^{j k} p_{T}^{j} S_{T}^{k}}{M_{N}} e_{T}^{\perp}\right] \\
\frac{1}{2} \operatorname{tr}\left[i \gamma_{5} \phi\left(x, \vec{p}_{T}\right)\right] & =\frac{M_{N}}{P^{+}}\left[S_{L} e_{L}+\frac{\vec{p}_{T} \vec{S}_{T}}{M_{N}} e_{T}\right] \\
\frac{1}{2} \operatorname{tr}\left[\gamma^{\alpha} \phi\left(x, \vec{p}_{T}\right)\right] & =\frac{M_{N}}{P^{+}}\left[\frac{p_{T}^{j}}{M_{N}} f^{\perp}+\varepsilon^{j k} S_{T}^{k} f_{T}+S_{L} \varepsilon^{j k} S_{T}^{k} f_{L}^{\perp}+\frac{\left(p_{T}^{j} p_{T}^{k}-\frac{1}{2} \vec{p}_{T}^{2} \delta^{j k}\right) \varepsilon^{k l} S_{T}^{l}}{M_{N}^{2}} f_{T}^{\perp}\right] \\
\frac{1}{2} \operatorname{tr}\left[\gamma^{j} \gamma_{5} \phi\left(x, \vec{p}_{T}\right)\right] & =\frac{M_{N}}{P^{+}}\left[S_{T}^{j} g_{T}+S_{L} \frac{p_{T}^{j}}{M_{N}} g_{L}^{\perp}+\frac{\left(p_{T}^{j} p_{T}^{k}-\frac{1}{2} \vec{p}_{T}^{2} \delta^{j k}\right) S_{T}^{k}}{M_{N}^{2}}+\frac{\varepsilon^{j k} p_{T}^{k}}{M_{N}} g^{\perp}\right] \\
\frac{1}{2} \operatorname{tr}\left[i \sigma^{j k} \gamma_{5} \phi\left(x, \vec{p}_{T}\right)\right] & =\frac{M_{N}}{P^{+}}\left[\frac{S_{T}^{j} p_{T}^{k}-S_{T}^{k} p_{T}^{j}}{M_{N}} h_{T}^{\perp}-\varepsilon^{j k} h\right], \\
\frac{1}{2} \operatorname{tr}\left[i \sigma^{+-} \gamma_{5} \phi\left(x, \vec{p}_{T}\right)\right] & =\frac{M_{N}}{P^{+}}\left[S_{L} h_{L}+\frac{\vec{p}_{T} \cdot \vec{S}_{T}}{M_{N}} h_{T}\right],
\end{aligned}
$$

where the space-indices $j, k$ refer to the plane transverse with respect to the light-cone and $\varepsilon^{12}=-\varepsilon^{21}=1$ and zero else. Integrating out transverse momenta in the correlator (1) leads to the 'usual' parton distributions known from collinear kinematics $j^{a}(x)=\int \mathrm{d}^{2} \vec{p}_{T} j^{a}\left(x, \vec{p}_{T}^{2}\right)$ with $j=f_{1}, g_{1}, h_{1}, e, g_{T}, h_{L}$ [102, 103]. Dirac-structures other than that in Eqs. (210) lead to subsubleading-twist terms 28].

For convenience we introduce for a generic TMD $j^{q}\left(x, k_{\perp}\right)$ the '(unintegrated) transverse (1)-moments' defined as

$$
j^{(1) q}\left(x, k_{\perp}\right)=\frac{k_{\perp}^{2}}{2 M_{N}^{2}} j^{q}\left(x, k_{\perp}\right), \quad j^{(1) q}(x)=\int \mathrm{d}^{2} k_{\perp} \frac{k_{\perp}^{2}}{2 M_{N}^{2}} j^{q}\left(x, k_{\perp}\right) .
$$

Moreover, we shall also make use of the '(1/2)-moments' defined for a generic TMD as

$$
f_{1}^{(1 / 2) q}(x)=\int \mathrm{d}^{2} k_{\perp} \frac{k_{\perp}}{2 M_{N}} f_{1}^{q}\left(x, k_{\perp}\right) .
$$




\section{TMDS IN THE BAG MODEL}

In the MIT bag model, the quark field has the following general form [104 106],

$$
\Psi_{\alpha}(\vec{x}, t)=\sum_{n>0, \kappa= \pm 1, m= \pm 1 / 2} N(n \kappa)\left\{b_{\alpha}(n \kappa m) \psi_{n \kappa j m}(\vec{x}, t)+d_{\alpha}^{\dagger}(n \kappa m) \psi_{-n-\kappa j m}(\vec{x}, t)\right\}
$$

where $b_{\alpha}^{\dagger}$ and $d_{\alpha}^{\dagger}$ create quark and anti-quark excitations in the bag with the wave functions

$$
\psi_{n,-1, \frac{1}{2} m}(\vec{x}, t)=\frac{1}{\sqrt{4 \pi}}\left(\begin{array}{r}
i j_{0}\left(\frac{\omega_{n,-1}|\vec{x}|}{R_{0}}\right) \chi_{m} \\
-\vec{\sigma} \cdot \hat{x} j_{1}\left(\frac{\omega_{n,-1}|\vec{x}|}{R_{0}}\right) \chi_{m}
\end{array}\right) e^{-i \omega_{n,-1} t / R_{0}} .
$$

For the lowest mode, we have $n=1, \kappa=-1$, and $\omega_{1,-1} \approx 2.04$ denoted as $\omega \equiv \omega_{1,-1}$ in the following. In the above equation, $\vec{\sigma}$ is the $2 \times 2$ Pauli matrix, $\chi_{m}$ the Pauli spinor, $R_{0}$ the bag radius, $\hat{x}=\vec{x} /|\vec{x}|$, and $j_{i}$, are spherical Bessel functions. Taking the Fourier transformation, we have the momentum space wave function for the lowest mode,

$$
\varphi_{m}(\vec{k})=i \sqrt{4 \pi} N R_{0}^{3}\left(\begin{array}{r}
t_{0}(k) \chi_{m} \\
\vec{\sigma} \cdot \hat{k} t_{1}(k) \chi_{m}
\end{array}\right)
$$

where $\hat{k}=\vec{k} / k$ with $k=|\vec{k}|$ and the normalization factor $N$ is,

$$
N=\left(\frac{\omega^{3}}{2 R_{0}^{3}(\omega-1) \sin ^{2} \omega}\right)^{1 / 2}
$$

The two functions $t_{i}, i=0,1$ are defined as

$$
t_{i}(k)=\int_{0}^{1} u^{2} d u j_{i}\left(u k R_{0}\right) j_{i}(u \omega)
$$

From the above equations, we see that the bag model wave function Eq. (15) contains both $S$ and $P$ wave components. Especially, $t_{0}$ represents the $S$-wave component, whereas $t_{1}$ represents the $P$-wave component of the proton wave functions.

With the above wave functions, we can calculate all quark TMDs. For convenience we define the constant $A$, which will be common to all TMDs, and the momenta $k_{z}$ and $k$ as

$$
A=\frac{16 \omega^{4}}{\pi^{2}(\omega-1) j_{0}^{2}(\omega) M_{N}^{2}}, \quad k=\sqrt{k_{z}^{2}+k_{\perp}^{2}}, \quad k_{z}=x M_{N}-\omega / R_{0}, \quad \widehat{k}_{z}=\frac{k_{z}}{k}, \quad \widehat{M}_{N}=\frac{M_{N}}{k}
$$

where $M_{N}$ is the proton mass, and the bag radius is fixed such that $R_{0} M_{N}=4 \omega$. Moreover, we assume $S U(6)$ spin-flavor symmetry of the proton wave function, such that spin-independent TMDs of definite flavor are given in terms of respective 'flavor-less' expressions multiplied by a 'flavor factor' $N_{q}$, and spin-dependent TMDs of definite flavor follow from multiplying the respective 'flavor-less' expressions by a 'spin-flavor factor' $P_{q}$ with

$$
N_{u}=2, \quad N_{d}=1, \quad P_{u}=\frac{4}{3}, \quad P_{d}=-\frac{1}{3} .
$$

We recall that in the quark model formulated for a general (odd) number of colors $N_{c}$, these flavor factors are given by $N_{u}=\left(N_{c}+1\right) / 2$ and $N_{d}=\left(N_{c}-1\right) / 2$ while $P_{u}=\left(N_{c}+5\right) / 6$ and $P_{d}=\left(-N_{c}+1\right) / 6[107]$.

We mention that the MIT bag model gives rise also to antiquark distributions, but to unphysical ones, since $f_{1}^{\bar{q}}(x)<0$, which violates positivity. The TMDs receive non-vanishing support also from the regions $|x| \geq 1$. Though non-physical these contributions must be included when evaluating sum rules like $\int \mathrm{d} x f_{1}^{q}(x)=N_{q}$ or the momentum sum rule $\sum_{q} \int \mathrm{d} x x f_{1}^{q}(x)=1$, i.e. sum rules are satisfied only when integrating over the whole $x$-axis.

In literature it was discussed how to deal with these caveats, see for example [108]. In this work, we limit ourselves to the discussion of quark TMDs at $0 \leq x \leq 1$, which should not be confused with 'valence distributions', for example $f_{1 \text { val }}^{q}(x)=f_{1}^{q}(x)-f_{1}^{\bar{q}}(x)$. When discussing sum rules, however, integration over the whole $x$-axis is implied.

Since there are no explicit gluon degrees of freedom, T-odd TMDs vanish in this model [91]. In principle, one can simulate the effect of the gauge link, which is crucial in QCD for T-odd effects [22 24], for example by introducing 'one-gluon-exchange' [91, 92] or invoking instanton effects 93. In this work we shall not consider such extensions of the bag model, and restrict ourselves to the description of T-even distributions. 


\section{A. Results for TMDs in the bag model}

In the notation introduced above, the results for the T-even leading twist TMDs are given by

$$
\begin{aligned}
f_{1}^{q}\left(x, k_{\perp}\right) & =N_{q} A\left[t_{0}^{2}+2 \widehat{k}_{z} t_{0} t_{1}+t_{1}^{2}\right] \\
g_{1}^{q}\left(x, k_{\perp}\right) & =P_{q} A\left[t_{0}^{2}+2 \widehat{k}_{z} t_{0} t_{1}+\left(2 \widehat{k}_{z}^{2}-1\right) t_{1}^{2}\right] \\
h_{1}^{q}\left(x, k_{\perp}\right) & =P_{q} A\left[t_{0}^{2}+2 \widehat{k}_{z} t_{0} t_{1}+\widehat{k}_{z}^{2} t_{1}^{2}\right] \\
g_{1 T}^{\perp q}\left(x, k_{\perp}\right) & =P_{q} A\left[2 \widehat{M}_{N}\left(t_{0} t_{1}+\widehat{k}_{z} t_{1}^{2}\right)\right] \\
h_{1 L}^{\perp q}\left(x, k_{\perp}\right) & =P_{q} A\left[-2 \widehat{M}_{N}\left(t_{0} t_{1}+\widehat{k}_{z} t_{1}^{2}\right)\right] \\
h_{1 T}^{\perp q}\left(x, k_{\perp}\right) & =P_{q} A\left[-2 \widehat{M}_{N}^{2} t_{1}^{2}\right]
\end{aligned}
$$

and for the subleading twist TMDs we obtain

$$
\begin{aligned}
e^{q}\left(x, k_{\perp}\right) & =N_{q} A\left[t_{0}^{2}-t_{1}^{2}\right] \\
f^{\perp q}\left(x, k_{\perp}\right) & =N_{q} A\left[2 \widehat{M}_{N} t_{0} t_{1}\right] \\
g_{T}^{q}\left(x, k_{\perp}\right) & =P_{q} A\left[t_{0}^{2}-\widehat{k}_{z}^{2} t_{1}^{2}\right] \\
g_{L}^{\perp q}\left(x, k_{\perp}\right) & =P_{q} A\left[2 \widehat{M}_{N} \widehat{k}_{z} t_{1}^{2}\right] \\
g_{T}^{\perp q}\left(x, k_{\perp}\right) & =P_{q} A\left[2 \widehat{M}_{N}^{2} t_{1}^{2}\right] \\
h_{L}^{q}\left(x, k_{\perp}\right) & =P_{q} A\left[t_{0}^{2}+\left(1-2 \widehat{k}_{z}^{2}\right) t_{1}^{2}\right] \\
h_{T}^{\perp q}\left(x, k_{\perp}\right) & =P_{q} A\left[2 \widehat{M}_{N} t_{0} t_{1}\right] \\
h_{T}^{q}\left(x, k_{\perp}\right) & =P_{q} A\left[-2 \widehat{M}_{N} \widehat{k}_{z} t_{1}^{2}\right]
\end{aligned}
$$

In the following Sections we shall discuss these results in detail. 


\section{EQUALITIES AND INEQUALITIES AMONG TMDS}

In QCD all TMDs are independent functions. But in quark models, due to absence of gauge field degrees of freedom, certain relations among different TMDs appear which must be satisfied in any consistent relativistic quark model. We discuss these "model-independent" quark-model relations in Sec. IIIA Of course, depending on a quark model further relations may appear, and the bag model results (20,33) provide a nice illustration why this happens which is demonstrated in Secs. IIIB and IIIC in detail. In Sec. IIID we compare to results from other models. This comparison helps to establish to which extent which relations might be expected to be useful in nature. Sec. IIIE is devoted to the discussion of one particular relation.

\section{A. Relations valid in all quark models}

Certain relations among TMDs must be valid in any quark model of the nucleon lacking gluon degrees of freedom [100]. In such "no-gluon models" the absence of the Wilson-link implies that in the general Lorentz-decomposition of the unintegrated quark-correlator certain amplitudes do not appear, namely the $B_{i}$-amplitudes $(i=1,2, \ldots 20)$ in the notation of [28]. This gives rise to the following relations [17, 100]

$$
\begin{aligned}
& g_{T}(x) \stackrel{\text { LIR }}{=} g_{1}(x)+\frac{\mathrm{d}}{\mathrm{d} x} g_{1 T}^{\perp(1)}(x), \\
& h_{L}(x) \stackrel{\text { LIR }}{=} h_{1}(x)-\frac{\mathrm{d}}{\mathrm{d} x} h_{1 L}^{\perp(1)}(x), \\
& h_{T}(x) \stackrel{\text { LIR }}{=}-\frac{\mathrm{d}}{\mathrm{d} x} h_{1 T}^{\perp(1)}(x), \\
& g_{L}^{\perp}(x)+\frac{\mathrm{d}}{\mathrm{d} x} g_{T}^{\perp(1)}(x) \stackrel{\text { LIR }}{=} 0, \\
& h_{T}\left(x, p_{T}\right)-h_{T}^{\perp}\left(x, p_{T}\right) \stackrel{\text { LIR }}{=} h_{1 L}^{\perp}\left(x, p_{T}\right),
\end{aligned}
$$

which must hold in any consistent relativistic quark model. These so-called "Lorentz-invariance relations" (LIRs) are not valid in models with gauge field degrees of freedom [95] and in QCD [96]. The applications of LIRs in phenomenology were discussed in [99, 100]. There it was also shown, by exploring QCD equations of motion, that some LIRs hold in an approximation consisting of the neglect of quark-gluon-quark-correlator and current quark mass terms. Whether such an approximation is is justified in nature is, of course, a different question. For discussions of specific cases see [98 101, 109 113]. For quark model calculations, the practical application of the relations (34) 38) is immediate: they provide a valuable cross check for the numerical results.

In App. $\mathrm{A}$ we provide analytical proofs that the LIRs (34 38) are satisfied in the bag model. We also checked that the numerical results satisfy the LIRs, which provides a welcome cross-check for the numerical calculation. 


\section{B. Linear relations in bag model}

In the bag model, there are 9 linear relations among the 14 (twist-2 and 3) T-even TMDs, which can be written as follows

$$
\begin{gathered}
\mathcal{D}^{q} f_{1}^{q}\left(x, k_{\perp}\right)+g_{1}^{q}\left(x, k_{\perp}\right)=2 h_{1}^{q}\left(x, k_{\perp}\right) \\
\mathcal{D}^{q} e^{q}\left(x, k_{\perp}\right)+h_{L}^{q}\left(x, k_{\perp}\right)=2 g_{T}^{q}\left(x, k_{\perp}\right) \\
\mathcal{D}^{q} f^{\perp q}\left(x, k_{\perp}\right)=h_{T}^{\perp q}\left(x, k_{\perp}\right) \\
g_{1 T}^{\perp q}\left(x, k_{\perp}\right)=-h_{1 L}^{\perp q}\left(x, k_{\perp}\right) \\
g_{T}^{\perp q}\left(x, k_{\perp}\right)=-h_{1 T}^{\perp q}\left(x, k_{\perp}\right) \\
g_{L}^{\perp q}\left(x, k_{\perp}\right)=-h_{T}^{q}\left(x, k_{\perp}\right) \\
g_{1}^{q}\left(x, k_{\perp}\right)-h_{1}^{q}\left(x, k_{\perp}\right)=h_{1 T}^{\perp(1) q}\left(x, k_{\perp}\right) \\
g_{T}^{q}\left(x, k_{\perp}\right)-h_{L}^{q}\left(x, k_{\perp}\right)=h_{1 T}^{\perp(1) q}\left(x, k_{\perp}\right) \\
h_{T}^{q}\left(x, k_{\perp}\right)-h_{T}^{\perp q}\left(x, k_{\perp}\right)=h_{1 L}^{\perp q}\left(x, k_{\perp}\right)
\end{gathered}
$$

where the 'dilution factor' is defined as

$$
\mathcal{D}^{q}=\frac{P_{q}}{N_{q}}
$$

In the relations (45, 46) some TMDs need to be multiplied by the model-independent factor $k_{\perp}^{2} /\left(2 M_{N}^{2}\right)$, which is a 'legitimate linear operation' in our context (the meaning of that will be explained shortly). The '(unintegrated) transverse moments' are defined in Eq. (11).

Why are there 9 linear relations? In fact, naively, one could have expected even more relations, since all TMDs are expressed in terms of only two functions, $t_{0}$ and $t_{1}$ representing the contributions from the $\mathrm{S}$ and $\mathrm{P}$-wave components of the proton wave function, Eqs. (15 17). However, having linear relations in mind, the combinations $t_{0}^{2}, t_{0} t_{1}, t_{1}^{2}$ are to be considered as independent structures. But there are more independent structures than that. By inspecting Eqs. (20-33) we see that the actual number of linearly independent structures in the TMDs is 5, namely

$$
\begin{aligned}
& \left.\begin{array}{l}
\text { I. } t_{0}^{2} \\
\text { II. } \widehat{k}_{z} t_{0} t_{1} \\
\text { III. } t_{1}^{2}, \widehat{k}_{z}^{2} t_{1}^{2}
\end{array}\right\} \leftrightarrow\left\{\begin{array}{l}
f_{1}^{q}, g_{1}^{q}, h_{1}^{q}, h_{1 T}^{\perp q} \text { (twist 2) } \\
e^{q}, g_{T}^{q}, h_{L}^{q}, g_{T}^{\perp q} \text { (twist 3) }
\end{array} \Rightarrow\right. \text { relations (39, 40, 43, 45, 46) } \\
& \left.\begin{array}{l}
\text { IV. } t_{0} t_{1} \\
\text { V. } \widehat{k}_{z} t_{1}^{2}
\end{array}\right\} \leftrightarrow\left\{\begin{array}{ll}
g_{1 T}^{\perp q}, h_{1 L}^{\perp q} & \text { (twist 2) } \\
f^{\perp q}, g_{L}^{\perp q}, h_{T}^{\perp q}, h_{T}^{q} & \text { (twist 3) }
\end{array} \Rightarrow\right. \text { relations [41 42 44 47) }
\end{aligned}
$$

where we show respectively to which TMDs the different structures contribute. We observe that in some sense there are two 'disconnected subspaces': one is due to the structures I, II, III, and the other due to the structures IV, V.

The structures II and IV, $\widehat{k}_{z} t_{0} t_{1}$ and $t_{0} t_{1}$, are linearly independent, as there is no way of relating one with the other in a model-independent way. Indeed, in order to do this, one should multiply a TMD by a factor including $k_{z}$ which explicitly depends on parameters of the bag model, as is evident from Eq. (18), and we discard such a manipulation as a model-dependent operation. For the same reason the structures in III and V are linearly independent.

But $\widehat{k}_{z}^{2} t_{1}^{2}$ and $t_{1}^{2}$ in point III are linearly dependent: if we multiply $t_{1}^{2}$ (actually in relevant expressions $\widehat{M}_{N}^{2} t_{1}^{2}$ appears) by the model-independent factor $k_{\perp}^{2} / M_{N}^{2}$ and add $\widehat{k}_{z}^{2} t_{1}^{2}$ we obtain just $t_{1}^{2}$ which happens in Eqs. (45, 46). Clearly, the multiplication of TMDs by $k_{\perp}^{2} /\left(2 M_{N}^{2}\right)$ is a model-independent manipulation leading to transverse moments in (11).

To summarize, there are 5 linearly independent structures in the bag model and 14 T-even TMDs. This implies 9 linear relations, and Eqs. (39) 477) represent one way of writing these relations. These findings mean that one can choose, in the bag model, a basis of 5 linearly independent TMDs, and construct the other TMDs from this basis. 


\section{Non-linear relations in the bag model}

In Eq. (49) we found that the TMDs in the bag model form two independent 'subspaces.' Let us summarize:

$$
\begin{aligned}
& \text { subspace A (I, II, III): } \underbrace{f_{1}^{q} g_{1}^{q} h_{1}^{q} h_{1 T}^{\perp q}}_{\text {twist } 2} \underbrace{e^{q} g_{T}^{q} h_{L}^{q} g_{T}^{\perp q}}_{\text {twist } 3} \Rightarrow \text { relations (39, 40, 43, 45, 46), } \\
& \text { subspace B (IV, V): } \underbrace{g_{1 T}^{\perp q} h_{1 L}^{\perp q}}_{\text {twist 2 }} \underbrace{f^{\perp q} g_{L}^{\perp q} h_{T}^{\perp q} h_{T}^{q}}_{\text {twist } 3} \Rightarrow \text { relations (41, 42, 44, 47). }
\end{aligned}
$$

In other words, there is no linear relation which would transform TMDs from subspace A into TMDs in subspace B. However, there are non-linear relations which can do that, for example,

$$
\begin{aligned}
h_{1}^{q}\left(x, k_{\perp}\right) h_{1 T}^{\perp q}\left(x, k_{\perp}\right) & =-\frac{1}{2}\left[h_{1 L}^{\perp q}\left(x, k_{\perp}\right)\right]^{2}, \\
g_{T}^{q}\left(x, k_{\perp}\right) g_{T}^{\perp q}\left(x, k_{\perp}\right) & =\frac{1}{2}\left[g_{1 T}^{\perp q}\left(x, k_{\perp}\right)\right]^{2}-g_{1 T}^{\perp q}\left(x, k_{\perp}\right) g_{L}^{\perp q}\left(x, k_{\perp}\right) .
\end{aligned}
$$

The results are presented such that on the left-(right-)hand-sides (L(R)HS) only TMDs from subspace A (B) appear.

The Eqs. (51, 52) are independent in the sense that it is impossible to convert one into the other upon use of the linear relations (39) [47). In order to convince oneself of that, notice that on RHS of (51) $h_{1 L}^{\perp q} \in$ subspace B appears from which alone one cannot construct the TMDs $\in$ subspace B on RHS of (52): from $h_{1 L}^{\perp q}$ one obtains $g_{1 T}^{\perp q}$ via (42) but not $g_{L}^{\perp q}$. However, for example, $g_{1 T}^{\perp q}$ and $g_{L}^{\perp q}$ span a basis which allows to construct all TMDs $\in$ subspace B. Similarly one finds that from the over-complete set of TMDs on LHS of (51, 52) all TMDs $\in$ subspace A follow. To summarize, the non-linear relations (51, 52) are independent, and these are the only independent non-linear relations.

Of course, upon the use of the linear relations (39) 47) one could generate further non-linear relations. One advantage of the presentation (51, 52) is that they connect only chirally even, or only chirally odd TMDs.

With the 9 linear relations (39 47) and the 2 non-linear relations (51) 52) we find altogether 11 relations among 14 TMDs in the bag model. This may reflect that eventually all TMDs can be traced back to the free structures proportional to $t_{0}^{2}, t_{0} t_{1}, t_{1}^{2}$.

It should be noticed that in Secs. ЩIB and ЩIC we permitted only manipulations of the kind: adding TMDs, multiplying them, and forming (1)-moments. If one includes differentiation of TMDs one obtains additional relations, see Sec. IIIA and App. A. 


\section{How general are quark model relations among TMDs?}

The deeper reason, why in the bag model relations among TMDs appear, is ultimately related to Melosh rotations which connect longitudinal and transverse nucleon and quark polarization states in a Lorentz-invariant way [114].

An important issue, when observing relations among TMDs in a model, concerns their presumed validity beyond that particular model framework. For that it is instructive to compare first to other models. In fact, some of the relations (39,47) were discussed previously in literature in various models. Let us review briefly.

- Eq. (39): its $k_{\perp}$-integrated version was discussed in bag model in [103] and [115, 116] and in light-cone constituent models in [117]. The unintegrated version was discussed in bag and light-cone constituent models [80, 81].

- Eq. (40): its integrated version was observed in the bag model previously in [115].

- Eq. (42): was first observed in the spectator model of [79] and later also in light-cone constituent models [81] and the covariant parton model of Ref. [82].

- Eq. (44): was found in the spectator model of Ref. [79].

- Eq. (45): was first observed in the bag [80]. It is valid also in the spectator [79], light-cone constituent [81], and covariant parton [82] models.

- Eqs. 41, 43, 46, 47): are new in the sense of not having been mentioned previously in literature. But the latter 3 are satisfied by the spectator model results from [79].

- The non-linear relation (51), which connects all T-even, chirally-odd leading-twist TMDs was observed in the covariant parton model approach [82]. Eq. (52) was not discussed so far in literature.

The detailed comparison, in which models these relations hold and in which they are violated, gives some insight into the question to which extent these relations are model-dependent.

Let us discuss first Eqs. (39 41), which include the 'dilution factor' (48) and connect polarized and unpolarized TMDs. For these relations SU(6)-spin-flavor symmetry is necessary, but not sufficient. In fact, this type of relations holds only in 'simplest models' such as the bag model version used here or light-cone constituent models [80, 81]. What these models have in common is that the nucleon wave-function is constructed from 'flavor-blind' quark wavefunctions multiplied by appropriate spin-flavor factors in Eq. (19). The SU(6) symmetry, however, does not need to be realized in a model that simply. For example, the spectator model of 79] is SU(6) symmetric. But it does not support (39 41) which are spoiled by the different masses of the (scalar and axial-vector) spectator diquark systems. Interestingly, it is possible to recover these relations in [79] in the limiting case of the scalar and axial-vector diquark masses becoming equal (justified in large- $N_{c}$ limit). We mention that (39, 40) also are not supported in the covariant parton model approach of [82]. However, also in that approach it is possible to 'restore' these relations by introducing additional, restrictive assumptions, see 82 for a detailed discussion. We conclude that the relations (39) 41) require strong model assumptions. It is difficult to estimate to which extent such relations could be useful approximations in nature, though they could hold in the valence- $x$ region with an accuracy of (20-30)\%, see the comparison of similar $\mathrm{SU}(6)$ predictions and data for the polarized neutron and proton structure functions in [86].

From the point of view of model dependence, it is 'safer' [80] to compare relations which include only polarized or only unpolarized TMDs. We know no example for the latter, however, the relations (42 47) are of the former type. It is gratifying to observe that these relations are satisfied not only in the bag model, this work and 80], but also in the spectator model version of Ref. 79]. The relations among the leading twist TMDs, Eqs. (42, 45), hold also in light-cone constituent [81], and covariant parton [82] models. They are also valid in the non-relativistic model [82]. Though they do not prove anything, these observations indicate that such relations could be valid in a wider class of quark models.

Of course, quark model relations among TMDs have limitations, even in quark models. In [85] various versions of spectator models were used, and in some versions the relations were not supported (42. 45). It is instructive to observe that also the quark-target model [89] not supported the relations [42] 45). In fact, the inclusion of gauge fields brings us a step closer to QCD as compared to quark models, at least in what concerns the involved degrees of freedom. Finally, in QCD none of such relations is valid, and all TMDs are independent structures.

It would be interesting to 'test' such quark model relations in other models, lattice QCD, and in experiment. The latter, in fact, provide a test for the usefulness of quark models themselves — or, more precisely, their application to TMDs. 


\section{E. A special linear relation among collinear functions}

By taking linear combinations of (39 47) one can obtain many more linear relations. It is worth to discuss in some more detail one particularly interesting relation, which can be obtained in this way. By eliminating the transverse moment of the pretzelosity distribution from Eqs. (45, 46), and integrating over transverse momenta, we obtain

$$
g_{1}^{q}(x)-h_{1}^{q}(x)=g_{T}^{q}(x)-h_{L}^{q}(x) .
$$

This relation holds also in its unintegrated form. There are several reasons, why this relation is interesting.

First, it involves only collinear parton distribution functions, which is the only relation of such type in bag model. (Actually (39, 40) are also of such type, but they include the 'dilution factor' and are supported only in models with simplest spin-flavor structures, see Sec. IIID.) The QCD evolution equation for all these functions are known, and they are different, which shows the limitation of this relation: even if for some reason (53) was valid in QCD at a certain renormalization scale $\mu_{0}$, it would break down at any other scale $\mu \neq \mu_{0}$. This is by no means surprising, and we expect such limitations for all model relations.

Second, for the first Mellin moment this relation is valid model-independently. Hereby we strictly speaking presume the validity of the Burkardt-Cottingham sum rule, which is equivalent to the statement $\int \mathrm{d} x g_{T}^{q}(x)=\int \mathrm{d} x g_{1}^{q}(x)$, and an analog sum rule for $h_{L}^{q}(x)$ and $h_{1}^{q}(x)$. In QCD there are doubts especially concerning the validity of the BurkardtCottingham sum rule. However, it is valid in many models such as bag [103] or chiral quark soliton model [118].

Third, though it certainly is not exact in QCD, it would be interesting to learn whether (53) is satisfied in nature approximately. Also this relation can be tested on the lattice, especially for low Mellin moments and in the flavour non-singlet case. Lattice QCD calculations for Mellin moments of $g_{T}^{q}(x)$ were reported in [12].

Forth, the relation (53) can be tested in models where collinear parton distribution functions were studied. Some results can be found in literature. For example, calculations of parton distribution functions in bag models [103, 115] support this relation (the bag model version of [103] coincides with the one used here). Moreover, the spectator model [79] supports this relation: it is equivalent to $g_{2}^{q}(x)=\frac{1}{2} h_{2}^{q}(x)$ in the notation of [79], and also the unintegrated version of (53) is valid there. One counter-example is known though: the chiral quark-soliton model does not support this relation [118, 126]. This observation could provide a hint in which models (53) is valid. The models where (53) holds include only the components in the nucleon wave-function with the quark orbital angular momenta up to $L=0,1,2$ at most. The chiral quark soliton model, which does not support (53), contains all quark angular momenta $L=0,1,2,3,4, \ldots$ but this point deserves further investigation.

Fifth, an important aspect of model relations is that they inspire interpretations. The relation (53) means that the difference between $g_{T}^{q}$ and $h_{L}^{q}$ is to the same extent a 'measure of relativistic effects in the nucleon' as the difference between helicity and transversity [103]. Both these differences are related to the transverse moment of pretzelosity, see Eqs. [45, 46) and [80].

\section{F. Inequalities}

Finally, we discuss inequalities among leading twist TMDs, which are valid in QCD and all models [21]

$$
\begin{array}{r}
f_{1}^{q}\left(x, k_{\perp}\right) \geq 0, \quad\left|g_{1}^{q}\left(x, k_{\perp}\right)\right| \leq f_{1}^{q}\left(x, k_{\perp}\right), \quad\left|h_{1}^{q}\left(x, k_{\perp}\right)\right| \leq f_{1}^{q}\left(x, k_{\perp}\right), \\
\left|h_{1}^{q}\left(x, k_{\perp}\right)\right| \leq \frac{1}{2}\left(f_{1}^{q}\left(x, k_{\perp}\right)+g_{1}^{q}\left(x, k_{\perp}\right)\right), \\
\left|h_{1 T}^{\perp q}\left(x, k_{\perp}\right)\right| \leq \frac{1}{2}\left(f_{1}^{q}\left(x, k_{\perp}\right)-g_{1}^{q}\left(x, k_{\perp}\right)\right), \\
g_{1 T}^{\perp(1) q}\left(x, k_{\perp}\right)^{2}+f_{1 T}^{\perp(1) q}\left(x, k_{\perp}\right)^{2} \leq \frac{k_{\perp}^{2}}{4 M_{N}^{2}}\left(f_{1}^{q}\left(x, k_{\perp}\right)^{2}-g_{1}^{q}\left(x, k_{\perp}\right)^{2}\right), \\
h_{1 L}^{\perp(1) q}\left(x, k_{\perp}\right)^{2}+h_{1}^{\perp(1) q}\left(x, k_{\perp}\right)^{2} \leq \frac{k_{\perp}^{2}}{4 M_{N}^{2}}\left(f_{1}^{q}\left(x, k_{\perp}\right)^{2}-g_{1}^{q}\left(x, k_{\perp}\right)^{2}\right),
\end{array}
$$

where we have to keep in mind that in the present quark model framework the inequalities simplify, due the absence of the T-odd TMDs $f_{1 T}^{\perp q}$ and $h_{1}^{\perp q}$. In App. B we demonstrate explicitly that the bag model expressions for the quark TMDs satisfy (54 58). It is interesting to remark that for the nucleon, except for $f_{1}^{q}\left(x, k_{\perp}\right) \geq 0$, all the other inequalities in (54 58) are 'true' (i.e. never saturated) inequalities, see App. B for a detailed discussion. 


\section{PRETZELOSITY AND QUARK ORBITAL ANGULAR MOMENTUM}

In quark models, in contrast to gauge theories, one may unambiguously define the quark orbital angular momentum operator as $\hat{L}_{q}^{i}=\bar{\psi}_{q} \varepsilon^{i k l} \hat{r}^{k} \hat{p}^{l} \psi_{q}$ where for clarity the 'hat' indicates a quantum operator. This definition follows (in the absence of gauge fields) uniquely, for instance, from identifying that part of the generator of rotations not associated with the intrinsic quark spin. For the following it will be convenient to introduce a 'non-local version' of this operator, by defining (keep in mind that we work in non-gauge theory)

$$
\hat{L}_{q}^{i}(0, z)=\bar{\psi}_{q}(0) \varepsilon^{i k l} \hat{r}^{k} \hat{p}^{l} \psi_{q}(z)
$$

In the bag model it is convenient to work in momentum space where $\hat{r}^{k}=i \frac{\partial}{\partial p^{k}}$ and $\hat{p}^{l}=p^{l}$. Next let us define, in analogy to Eq. (11) the following quantity

$$
L_{q}^{j}\left(x, p_{T}\right)=\left.\int \frac{\mathrm{d} z^{-} \mathrm{d}^{2} \vec{z}_{T}}{(2 \pi)^{3}} e^{i p z}\left\langle N\left(P, S^{3}\right)\left|\hat{L}_{q}^{i}(0, z)\right| N\left(P, S^{3}\right)\right\rangle\right|_{z^{+}=0, p^{+}=x P^{+}} .
$$

In order to find a connection to TMDs we must consider a longitudinally polarized nucleon, choosing the polarization vector as $S=(0,0,1)$ for definiteness, and we must focus on the $j=3$ component in (60), i.e. on the component of the angular momentum operator along the light-cone space-direction. This is because the transverse momenta $\vec{p}_{T}$ of the quarks generate orbital angular momentum which is oriented perpendicular to $\vec{p}_{T}$ (and to the transverse position of quarks inside the nucleon, which can be quantified rigorously in the impact parameter space in terms of generalized parton distribution functions, but we do not need this notion here).

In a quark model, where the ambiguities of gauge field theories are absent, the partonic interpretation of (60) is the following. For example, in a longitudinally polarized nucleon $L_{q}^{3}\left(x, p_{T}\right) \mathrm{d}^{2} \vec{p}_{T} \mathrm{~d} x$ tells how much the orbital angular momentum of a quark of flavour $q$, which carries the longitudinal momentum fraction $x$ and the transverse momentum $p_{T}=\left|\vec{p}_{T}\right|$, contributes to the nucleon spin. (In QCD such an interpretation for the light-cone plus-component $L_{q}^{+}$ would also be possible, in an appropriately fixed gauge.)

Evaluating the expression (60) in the bag model we obtain

$$
L_{q}^{3}\left(x, p_{T}\right)=(-1) h_{1 T}^{\perp(1) q}\left(x, p_{T}\right) .
$$

In order to demonstrate the consistency of this result we compute the contribution to the total angular momentum of the nucleon $J_{q}^{3}$ due to flavour $q . J_{q}^{3}$ is composed of contributions from intrinsic quark spin, $S_{q}^{3}=\frac{1}{2} \int \mathrm{d} x g_{1}^{q}(x)$, and quark orbital angular momentum $L_{q}^{3}=\int \mathrm{d} x \int \mathrm{d}^{2} \vec{p}_{T} L_{q}^{3}\left(x, p_{T}\right)$. We obtain

$$
\begin{aligned}
2 J_{q}^{3} & =\int \mathrm{d} x \int \mathrm{d}^{2} k_{\perp}\left[g_{1}^{q}\left(x, k_{\perp}\right)-2 h_{1 T}^{\perp(1) q}\left(x, k_{\perp}\right)\right] \\
& =P_{q} \frac{A}{M_{N}} \int \mathrm{d}^{3} k\left[t_{0}^{2}+2 \widehat{k}_{z} t_{0} t_{1}+\left(2 \widehat{k}_{z}^{2}-1+2 \frac{k_{\perp}^{2}}{k^{2}}\right) t_{1}^{2}\right] \\
& =P_{q} \frac{A}{M_{N}} \int \mathrm{d}^{3} k\left[t_{0}^{2}+t_{1}^{2}\right] \\
& =P_{q}
\end{aligned}
$$

where we first substituted $x \rightarrow k_{z} \equiv x M_{N}-\omega / R$ (recalling that $x$-integration is carried over entire $x$-axis, Sec. II), then used that under the integral over $\mathrm{d}^{3} k$ for symmetry reasons $\widehat{k}_{z}=k_{z} / k$ drops out while $k_{z}^{2} \rightarrow \frac{1}{3} k^{2}$ and $k_{\perp}^{2} \rightarrow \frac{2}{3} k^{2}$, and finally observed the same integral which appears in the normalization of the unpolarized distribution.

Eq. (62) is the correct SU(6) quark model result for the contributions of various flavours to the total nucleon spin. Notice that $J_{u}^{3}+J_{d}^{3}=\frac{1}{2}$. This confirms that the connection of pretzelosity and the quark orbital angular momentum content of the nucleon is consistent. Thus, our results, supported by the light-cone SU(6) quark-diquark model [84], suggest that

$$
L_{q}^{3}=(-1) \int \mathrm{d} x h_{1 T}^{\perp(1) q}(x)
$$

It is important to observe that the relation of pretzelosity and orbital angular momentum, Eqs. (61) and (63), is at the level of matrix elements of operators, and there is no a priori operator identity which would make such a connection. 


\section{NUMERICAL RESULTS}

In this Section we discuss the numerical results for the TMDs. In Sec. VA we present the results for the integrated TMDs as functions of $x$. In Sec. $\nabla B$ we focus on their $k_{\perp}$-behaviour. Finally, in Sec. $\nabla C$ we investigate the question whether the bag model results support the so-called Wandzura-Wilczek(-type) approximations.

\section{A. Results for the integrated TMDs}

As the flavour dependence governed by the spin-flavour SU(6) symmetry is trivial, we will show only results for the $u$-flavor. For unpolarized TMDs the $d$-quark distributions are factor 2 smaller than the $u$-quark distributions. In the case of the polarized TMDs, the $d$-quark distributions are factor 4 smaller and have opposite sign compared to the $u$-quark distributions, according to Eq. (19). All results discussed below refer to the low scale of the bag model.

Let us start the discussion of the numerical results with unpolarized TMDs. Fig. 19 shows the twist-2 unpolarized distribution function $f_{1}^{u}(x)$, and the subleading twist functions $f^{\perp u}(x), e^{u}(x)$. Only $f^{\perp q}(x)=\int \mathrm{d}^{2} k_{\perp} f^{\perp q}\left(x, k_{\perp}\right)$ is new in this figure. The remarkable observation is that $f^{\perp q}(x)$ is rather large, even larger than $f_{1}^{q}(x)$ for $x \lesssim 0.7$. However, one has to keep in mind that there are no positivity bounds for twist-3 TMDs. Moreover, it is $\frac{k_{\perp}}{M} f^{\perp q}\left(x, k_{\perp}\right)$ which enters in cross sections, and typically $\left\langle k_{\perp}\right\rangle \ll M_{N}$, which eventually guarantees positivity of cross sections. We remark that QCD equations of motion [114] imply a $\delta(x)$-contribution in $e^{q}(x)$, which is found in some [119, 120] but not all effective approaches [79, 121], including the bag model, see [103, 115] and Fig. 17.

Fig. 10 shows the polarized functions $g_{T}^{\perp u}(x), g_{1 T}^{\perp u}(x), h_{1}^{u}(x), g_{1}^{u}(x)$. The TMDs $h_{1 T}^{\perp a}(x)$ and $h_{1 L}^{\perp a}(x)$ are simply related to the shown TMDs according to $h_{1 T}^{\perp a}(x)=-g_{T}^{\perp a}(x)$ and $h_{1 L}^{\perp a}(x)=-g_{1 T}^{\perp a}(x)$, such that the results for these TMDs do not need to be shown. Also the results for $d$-quark distributions are not shown, as explained above. The results for the TMDs $g_{T}^{\perp a}(x), g_{1 T}^{\perp a}(x), h_{1 L}^{\perp a}(x), h_{1 T}^{\perp a}(x)$ are new, and it is interesting to observe that they are rather sizable, but again there are no positivity constraints on these objects.

Fig. 15 shows the polarized functions $g_{L}^{\perp u}(x), g_{T}^{\perp u}(x), h_{L}^{u}(x), g_{T}^{u}(x)$. The TMD $h_{T}^{u}(x)$ is not shown, being related to $g_{L}^{\perp u}(x)$ as $h_{T}^{u}(x)=-g_{L}^{\perp u}(x)$. We see that $h_{T}^{\perp u}$ is rather sizable, it is even bigger than $f_{1}^{u}(x)$ (the same scale is used in Figs. 1 $\mathrm{a}-\mathrm{c})$. Again there is no positivity constraint for this TMD, which would object this.

The large size of the integrated twist-2 TMDs $g_{1 T}^{\perp a}(x), h_{1 L}^{\perp a}(x), h_{1 T}^{\perp a}(x)$ can be understood qualitatively in the nonrelativistic limit which was formulated for an arbitrary number of colours $N_{c}$ in [82], and can eventually be traced back the convention of using the nucleon mass $M_{N}$ in order to compensate the dimension of the $k_{\perp}$ factor(s) in the decomposition of the correlators in Eq. (2) 10).

It is interesting to notice that $g_{L}^{\perp q}(x)=-h_{T}^{q}(x)$ are the only TMDs in the bag model which have a zero in the valence- $x$ region. This observation is actually not surprising but a consequence of the fact that the LIRs (36, 37) hold, and $g_{T}^{\perp(1) q}(x)=-h_{1 T}^{\perp(1) q}(x)$ have extrema in the valence- $x$ region.
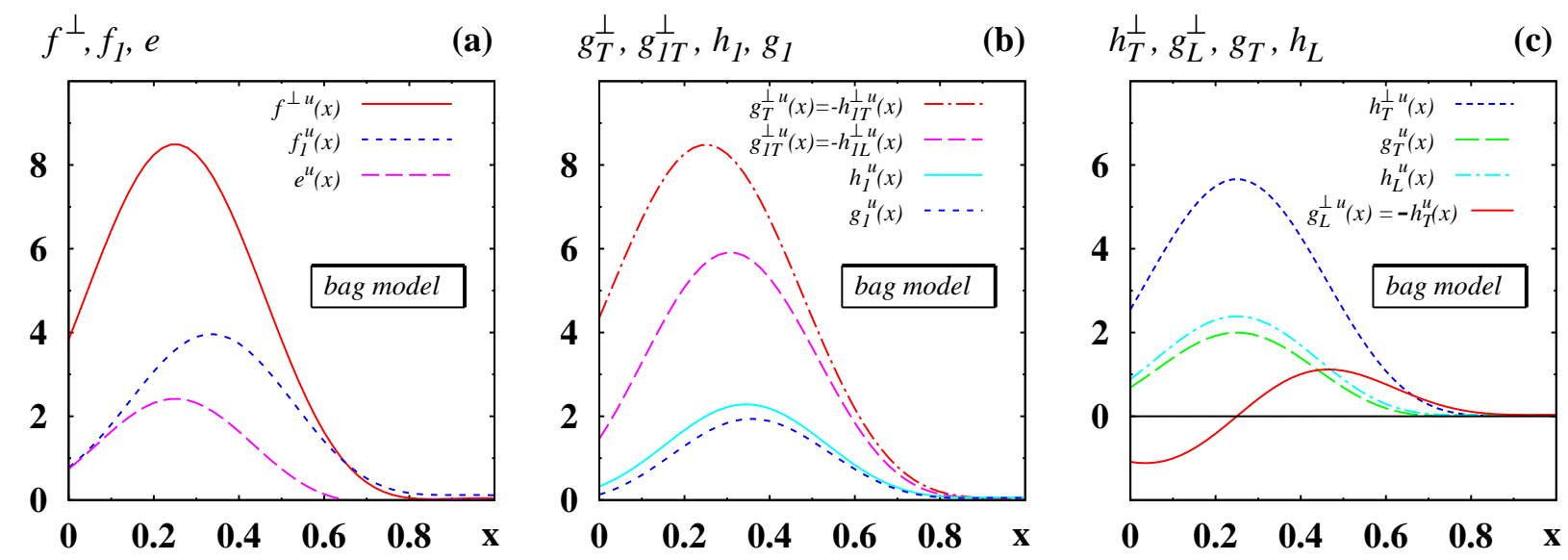

FIG. 1: (a) The unpolarized functions $f^{\perp u}(x), f_{1}^{u}(x), e^{u}(x)$ vs. $x$ from the bag model at the low scale. The $d$-quark distributions are factor two smaller compared to the unpolarized $u$-quark distributions according to the SU(6)-flavour factors in Eqs. (19) 27). (b) The polarized functions $g_{T}^{\perp u}(x)=-h_{1 T}^{\perp u}(x), g_{1 T}^{\perp u}(x)=-h_{1 L}^{\perp u}(x), h_{1}^{u}(x), g_{1}^{u}(x)$ vs. $x$. The $d$-quark distributions are factor four smaller and have opposite sign compared to the $u$-quark distributions according to the SU(6)-flavour factors in Eqs. (19) 27). (c) The polarized functions $h_{T}^{\perp u}(x), g_{L}^{\perp u}(x)=-h_{T}^{u}(x), g_{T}^{u}(x), h_{L}^{u}(x)$ vs. $x$. The $d$-quark functions are as in (b). 


\section{B. Transverse momentum dependence}

In the context of TMDs the most interesting aspect is, of course, their transverse momentum dependence. In principle, all information is contained in the two-dimensional functions $j\left(x, k_{\perp}\right)$ for a generic TMD, but here we shall content ourselves to discuss 'one- or zero-dimensional' projections of that information.

The first point we address is: what are the typical transverse momenta of unpolarized quarks in the bag TMDs? For that we define for a generic TMD $j^{q}\left(x, k_{\perp}\right)$ the following quantities

$$
\left\langle p_{T}\right\rangle=\frac{\int \mathrm{d} x \int \mathrm{d}^{2} k_{\perp} k_{\perp} j^{q}\left(x, k_{\perp}\right)}{\int \mathrm{d} x \int \mathrm{d}^{2} k_{\perp} j\left(x, k_{\perp}\right)}, \quad\left\langle p_{T}^{2}\right\rangle=\frac{\int \mathrm{d} x \int \mathrm{d}^{2} k_{\perp} k_{\perp}^{2} j^{q}\left(x, k_{\perp}\right)}{\int \mathrm{d} x \int \mathrm{d}^{2} k_{\perp} j\left(x, k_{\perp}\right)} .
$$

Due to the simple spin flavor structure of the MIT bag model the $\left\langle p_{T}\right\rangle$ and $\left\langle p_{T}^{2}\right\rangle$ are flavor-independent for all TMDs.

The first observation is that depending on the TMD $\left\langle p_{T}\right\rangle$ and $\left\langle p_{T}^{2}\right\rangle$ in Eq. (64) may not exist in the bag model, because the momentum-space wave-function components $t_{i}(k)$, Eq. (17), do not vanish sufficiently fast at large $k$. This is the case especially for $f_{1}^{q}\left(x, k_{\perp}\right)$.

For the same reason also the $(1)$-moment $f_{1}^{(1) q}(x)$ does not exist. However, the $(1 / 2)$-moment $f_{1}^{(1 / 2) q}(x)$ defined according to (12) exists, and can be used to introduce an $x$-dependent average transverse momentum $\left\langle p_{T}(x)\right\rangle$ as

$$
\left\langle p_{T}(x)\right\rangle=2 M_{N} \frac{f_{1}^{(1 / 2) q}(x)}{f_{1}^{q}(x)} .
$$

Fig. 2a shows the result for $f_{1}^{(1 / 2) q}(x)$. (The divergence of $\left\langle p_{T}\right\rangle$ from (64) emerges when one tries to integrate $f_{1}^{(1 / 2) q}(x)$ over $x$, recalling that this integration extends to the entire $x$-axis, see Sec. [I)

Now the (1)-moment $f_{1}^{(1) q}(x)$ is divergent, but its derivative with respect to $x$ exists, see the dotted line in Fig. 2 b. Hereby it is understood that the (1)-moment is computed with a finite cutoff $\Lambda_{\text {cut }} \gg M_{N}$, then the derivative is taken, and only then the limit $\Lambda_{\text {cut }} \rightarrow \infty$ is performed.

By integrating the well-defined $\frac{\mathrm{d}}{\mathrm{d} x} f_{1}^{(1) q}(x)$ we can compute a regularized (1)-moment $f_{1}^{(1) q}(x)_{\text {reg }}$. The result depends on some arbitrary integration constant, which we fix such that the (1)-moment vanishes at $x=1$. This choice is reasonable but not unique, if we recall that in the MIT bag model TMDs in general have a non-zero (though small) support for $|x| \geq 1$, see Sec. II. Our main conclusions in this respect, to be presented below in this Section, depend weakly on the chosen value of the integration constant, provided reasonable choices are made (such as, for example, $f_{1}^{(1) q}(x)_{\text {reg }}=f_{1}^{(1 / 2) q}(x)$ at $\left.x=1\right)$. The result for $f_{1}^{(1) q}(1)_{\text {reg }}$ defined in this way is shown as solid line in Fig. 20 b

With $f_{1}^{(1) q}(x)_{\text {reg }}$ we are in the position to define an $x$-dependent average transverse momentum square $\left\langle p_{T}^{2}(x)\right\rangle$ as

$$
\left\langle p_{T}^{2}(x)\right\rangle=2 M_{N}^{2} \frac{f_{1}^{(1) q}(x)_{r e g}}{f_{1}^{q}(x)} .
$$
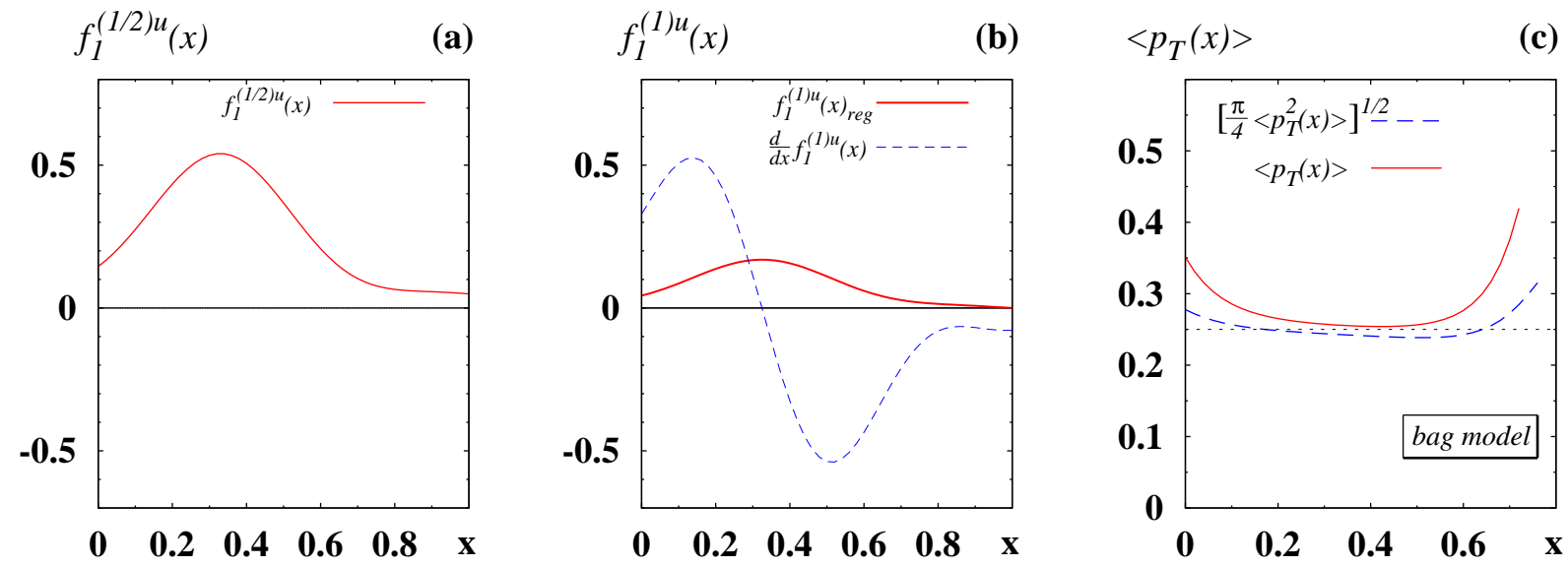

FIG. 2: For the unpolarized TMD $f_{1}^{q}\left(x, k_{\perp}\right)$ (a) the (1/2)-moment defined in Eq. (65), (b) the derivative of the (1)-moment and the regularized (1)-moment as discussed in the text, and (c) $\left\langle p_{T}(x)\right\rangle$ in comparison to $\left(\pi\left\langle p_{T}^{2}(x)\right\rangle / 4\right)^{1 / 2}$. In the Gauss-model the two quantities would be equal. (The dotted marks the value $\left\langle p_{T}(x)\right\rangle=0.25 \mathrm{GeV}$.) 
Fig. 22 shows $\left\langle p_{T}(x)\right\rangle$ as solid line. We observe that in the valence- $x$ region at the low hadronic scale $\left\langle p_{T}(x)\right\rangle$ very weakly depends on $x$. Numerically we find

$$
\left\langle p_{T}(x)\right\rangle \approx 0.25 \mathrm{GeV} \text { for } 0.2 \lesssim x \lesssim 0.5
$$

(The $\left\langle p_{T}(x)\right\rangle=0.25 \mathrm{GeV}$ is marked as dotted line in Fig. 2. .) This is similar to results from the light-cone constituent model [81] which also refer to a very low hadronic scale. In fact, keeping in mind the $p_{T}$-broadening effects due to gluon radiation with increasing normalization scale [11], this is a reasonable result at a low scale. (We remark that in parton model approaches one finds comparably low values for $\left\langle p_{T}(x)\right\rangle$ (albeit there the results refer to high scales) 122, 123 models.)

In phenomenology at high scales, however, larger values are required [58 60]. For example, the interpretations of SIDIS data from EMC [36] or HERMES [39] require

$$
\left\langle p_{T}(x)\right\rangle_{\text {Gauss }}= \begin{cases}0.64 \mathrm{GeV} & \text { from EMC data in [59], } \\ 0.56 \mathrm{GeV} & \text { from HERMES data in [60], }\end{cases}
$$

where the index "Gauss" indicates that the Gaussian model has been assumed in these studies. The Gaussian model means that $f_{1}^{q}\left(x, p_{T}\right)=f_{1}^{q}(x) \exp \left(-p_{T}^{2} /\left\langle p_{T}^{2}(x)\right\rangle_{\text {Gauss }}\right) /\left(\pi\left\langle p_{T}^{2}(x)\right\rangle_{\text {Gauss }}\right)$. The width $\left\langle p_{T}^{2}(x)\right\rangle_{\text {Gauss }}$ could be a function of $x$, but in practice it is often assumed to be a constant. Such an Ansatz works with sufficient precision for many practical applications in phenomenological studies [58 60]. In the Gaussian model the relation holds

$$
\left\langle p_{T}(x)\right\rangle_{\text {Gauss }}=\left[\frac{\pi}{4}\left\langle p_{T}^{2}(x)\right\rangle_{\text {Gauss }}\right]^{1 / 2} .
$$

Of course, in no model considered so far such a factorized $x$ - and transverse parton momentum dependence was ever observed, and in the bag model we do not observe it either. However, it is interesting to ask, for example, to which extent the relation (69) is supported in a model. With $\left\langle p_{T}^{2}(x)\right\rangle$ defined in (66) we obtain for the expression on the RHS of (69) the result plotted as dashed line in Fig. 2r. The remarkable observation is that (69) is supported within an accuracy of $\mathcal{O}(10 \%)$ in the valence- $x$ region. We remark that this conclusion is insensitive to the way the integration constant in $f_{1}^{(1) q}(x)_{\text {reg }}$ is fixed, provided this is done in a reasonable way (see above).

However, the bag model supports the Gaussian model much more than that, in the following sense. In the Gauss model we have $f_{1}^{q}\left(x, p_{T}\right)=f_{1}^{q}(x, 0) \exp \left(-p_{T}^{2} /\left\langle p_{T}^{2}(x)\right\rangle_{\text {Gauss }}\right)$ where, by definition, $f_{1}^{q}(x, 0)=f_{1}^{q}(x) /\left(\pi\left\langle p_{T}^{2}(x)\right\rangle_{\text {Gauss }}\right)$. When dealing with a model with non-Gauss-like transverse momentum dependence, this can be used to 'fit' the Gaussian width

$$
\left\langle p_{T}^{2}(x)\right\rangle_{\text {Gauss }}=\pi \frac{f_{1}^{q}(x, 0)}{f_{1}^{q}(x)}
$$

such that the Gaussian model is exact at $p_{T}=0$. By continuity arguments the Gaussian model can be expected to be a good approximation to the exact model results also for $p_{T}>0$ in some vicinity close to $p_{T}=0$. The question
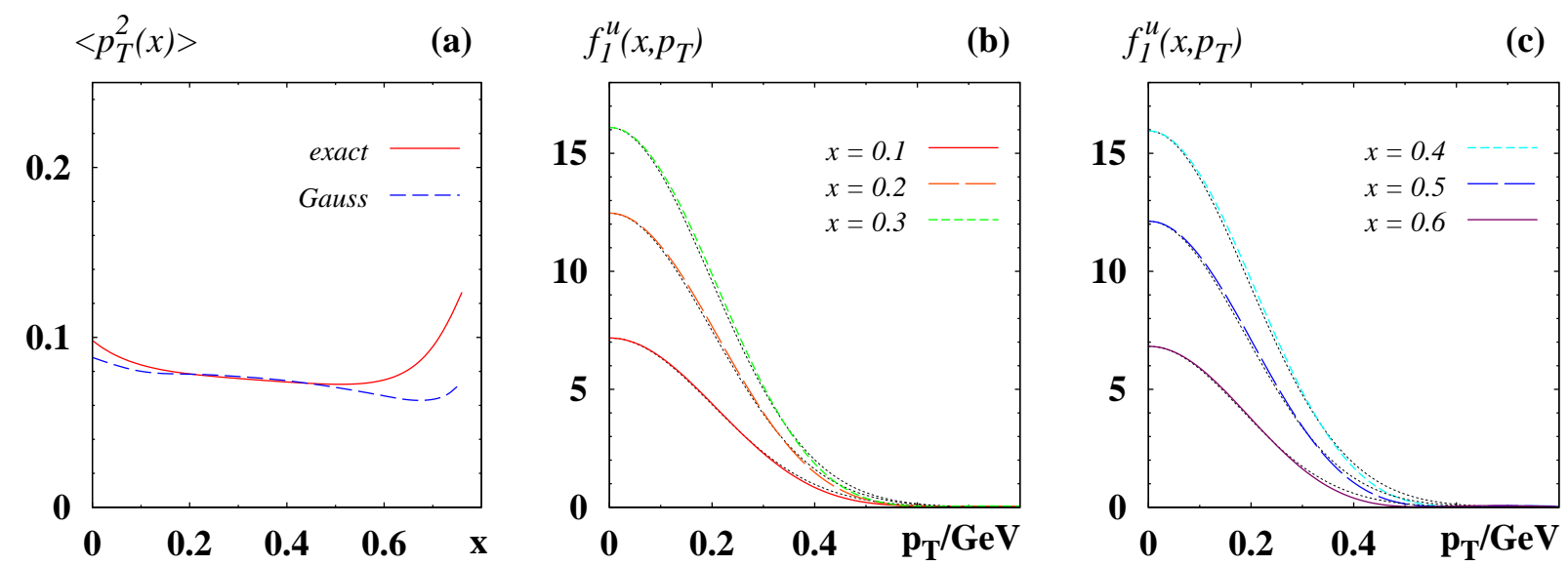

FIG. 3: (a) $\left\langle p_{T}^{2}\right\rangle$ of $f_{1}^{q}$ as function of $x$. Solid line: computed using the exact definition in Eq. (66). Dashed line: using the Gauss model relation, Eq. (70) . (b) and (c) $f_{1}^{q}\left(x, p_{T}\right)$ vs. $p_{T}$ for selected values of $x$. The thin dotted lines are the respective Gauss model approximations obtained from the Gaussian widths from Fig. 3a. 
is: how large is the $p_{T}$-region in which the Gaussian model with the width (70) will provide a useful approximation to the $p_{T}$-dependence of the unpolarized TMD?

Let us first compare the results for the mean transverse momentum square $\left\langle p_{T}^{2}(x)\right\rangle$ defined in Eq. (66) and the expression from the Gauss model $\left\langle p_{T}^{2}(x)\right\rangle_{\text {Gauss }}$, Eq. (70). It has to be noticed that these are a priori completely different quantities. It is therefore remarkable that the results agree so well, especially for valence $x$, see Fig. 3 .

In order to see to which extent the bag model is compatible with a Gauss-like shape of the transverse momentum distributions, we plot the $p_{T}$-dependence of $f_{1}^{u}\left(x, p_{T}\right)$ for $0 \leq p_{T}<M_{N}$ for selected values of $x=0.1,0.2,0.3$ in Fig. 3 $\mathrm{b}$, and $x=0.4,0.5,0.6$ in Fig. 35. In Figs. 3b and c, we also plot the respective Gaussian approximations (as thindotted lines). The result is astonishing: In the valence- $x$ region the exact curves and their Gaussian approximations agree excellently!

Not visible in Figs. $3 \mathrm{~b}$ and $\mathrm{c}$ is that the first worthwhile mentioning deviations from the Gaussian behaviour start to become apparent only at larger $p_{T}>0.5 \mathrm{GeV}$. The crucial difference is obviously in the large- $p_{T}$ asymptotics: $f_{1}^{q}\left(x, p_{T}\right) \sim \alpha\left(x, p_{T}\right) / p_{T}^{4}$ with $\left|\alpha\left(x, p_{T}\right)\right|<$ const. (The function $\alpha\left(x, p_{T}\right)$ oscillates, with a period defined by the periods of the spherical Bessel functions in (14), around some value which depends on the TMD but not on $x$. For all TMDs the respective functions $\alpha\left(x, p_{T}\right)$ are bound from above and below.)

Of course, non-perturbative models aiming at an effective description of the nucleon properties at hadronic scale are not able to address the large- $p_{T}$ region, where one may apply perturbative QCD. Effective models can, however, provide valuable insights for transverse momenta up to the order of magnitude of the hadronic scale, i.e. for $p_{T}<M_{N}$. In this $p_{T}$-region the bag model supports the concept of a Gaussian distribution of the transverse parton momenta in the case of $f_{1}^{q}\left(x, p_{T}\right)$.

Let us now turn our attention to other TMDs, keeping the discussion shorter after the detailed investigation of $f_{1}^{q}$. The TMDs $g_{1}^{q}, h_{1}^{q}, e^{q}, g_{T}, h_{L}^{q}$, which exist also as collinear parton distribution functions, have the same large- $p_{T}$ behavior like $f_{1}^{q}$, and consequently also have divergent (1)-moments (which can be regularized as in the case of $f_{1}^{q}$ ). In contrast to this $g_{1 T}^{\perp q}, h_{1 L}^{\perp q}, f^{\perp q}, h_{T}^{\perp q}$ behave like $\alpha\left(x, p_{T}\right) / p_{T}^{5}$ at large $p_{T}$ and have well-defined convergent (1)-moments. Finally $h_{1 T}^{\perp q}, g_{L}^{\perp q}, g_{T}^{\perp q}, h_{T}^{q}$ behave like $\alpha\left(x, p_{T}\right) / p_{T}^{6}$ and at large $p_{T}$ have well-defined convergent (1)-moments, too.

Is it possible to approximate the $p_{T}$-dependence also of the other TMDs in a Gaussian model? The answer is yes. Fig. 4 shows the Gaussian widths $\left\langle p_{T}^{2}(x)\right\rangle_{\text {Gauss }}$ as defined in Eq. (70) for the all TMDs. (Widths of $h_{1 L}^{\perp q}, f^{\perp q}, g_{T}^{\perp q}$ are not shown, because they correspond to those of respectively $g_{1 T}^{\perp q}, h_{T}^{\perp q}, h_{1 T}^{\perp q}$ thanks to the relations (41 43). Also the widths of $g_{L}^{\perp q}=-h_{T}^{q}$ are not shown, because of the discarded relation $g_{L}^{\perp q}=-k_{z} / M_{N} h_{1 T}^{\perp q}$ implies the same $p_{T}$-behaviour of $h_{1 T}^{\perp q}, g_{L}^{\perp q}, h_{T}^{q}$.)

We observe a modest $x$-dependence of the various $\left\langle p_{T}^{2}(x)\right\rangle_{\text {Gauss }}$, see Figs. 4 a-c. Important for the widths of $g_{1}^{q}, h_{1}^{q}$ is that they are not larger than that of $f_{1}^{q}$ in order to comply with positivity, which is of course the case, see Fig. 4ha. However, the widths of other TMDs are not bound in this way by $\left\langle p_{T}^{2}(x)\right\rangle_{\text {Gauss }}$ of $f_{1}^{q}$. In fact, the $\left\langle p_{T}^{2}(x)\right\rangle_{\text {Gauss }}$ of the pretzelosity distribution $h_{1 T}^{\perp q}$ exceeds the width of $f_{1}^{q}$, see Fig. 囷. Taken literally this would imply a violation of positivity, but we have to keep in mind that the $\left\langle p_{T}^{2}(x)\right\rangle_{\text {Gauss }}$ only approximate the true $p_{T}$-behavior of TMDS in the model, and the exact model results always satisfy positivity, see Sec. IIIF and App. B
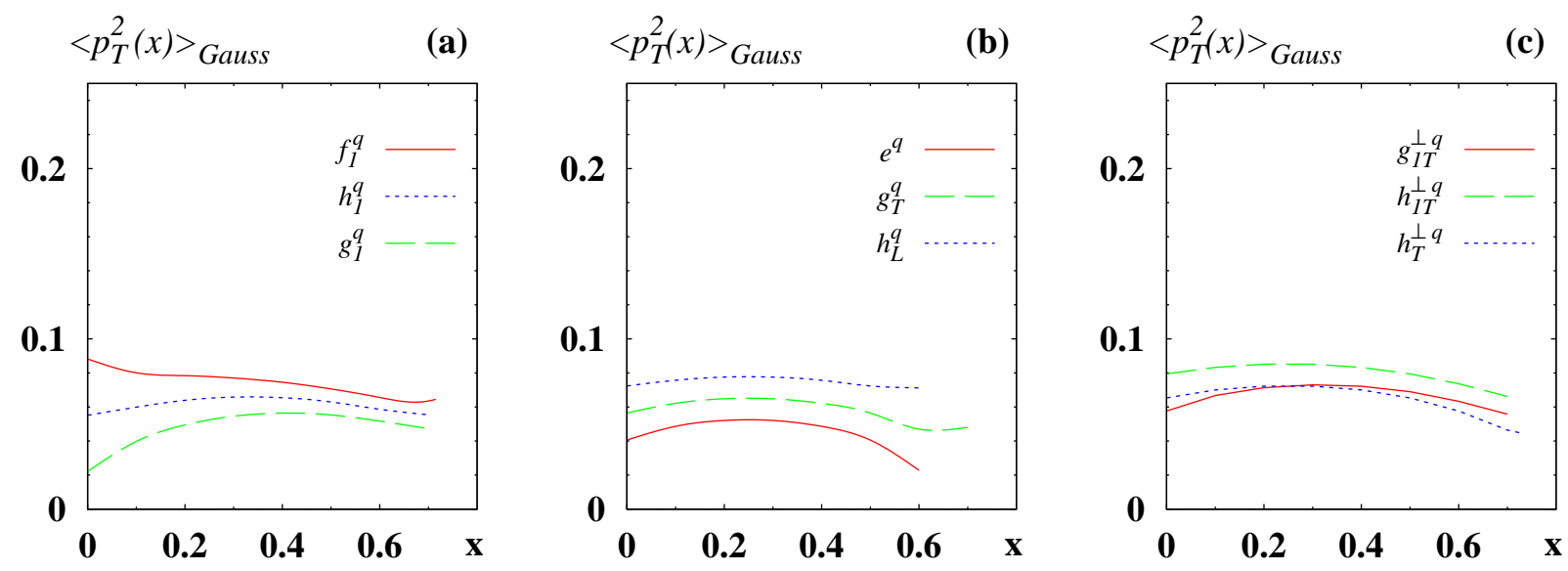

FIG. 4: The Gaussian widths as defined in Eq. (70) vs. $p_{T}$ for various TMDs. 
Fig. 5 shows the $p_{T}$-dependence of the TMDs for selected values of $x$, chosen to optimize the clarity of the plots. The thin dotted lines are the respective Gauss model approximations obtained from the Gaussian widths from Fig. 4 . We observe in general a good agreement, including even $g_{L}^{\perp} u$ which has a zero in $x$, see Fig. 4 .

Some TMDs, most notably for $g_{1}^{q}$ and $e^{q}$ in Fig. 4 a and 4 e, have for fixed values of $x$ a zero in $p_{T}$. One is tempted to suspect a model-artifact which can be traced back to the oscillatory behaviour of the Bessel functions in (14). However, in a covariant parton model calculation it was observed that $g_{1}^{u}\left(x, p_{T}\right)$ becomes negative for some values of $x, p_{T}$ [83].
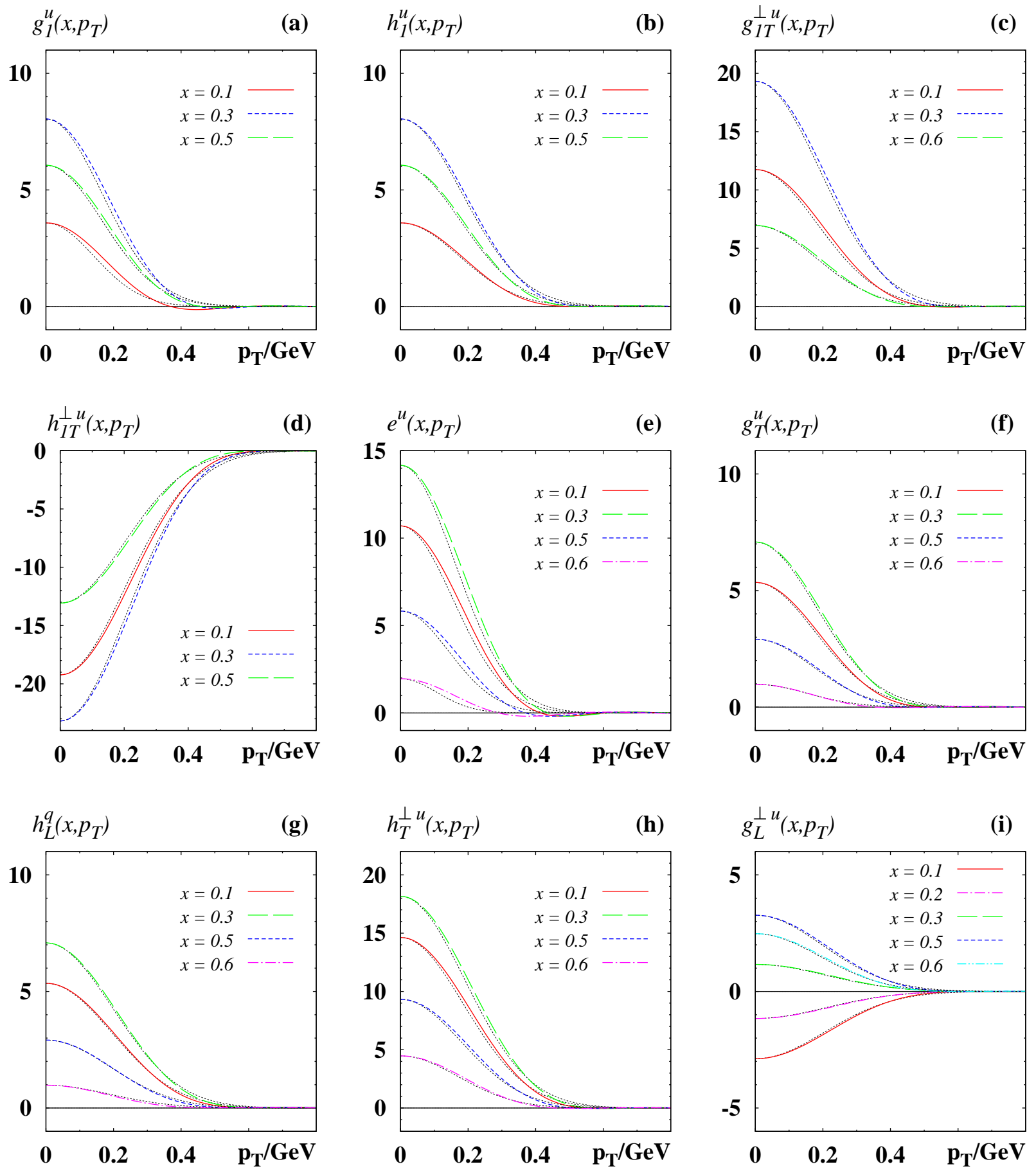

FIG. 5: The $p_{T}$-dependence of various TMDs for selected values of $x$. The thin dotted lines are the respective Gauss model approximations obtained from the Gaussian widths from Fig. 4 
This detail may deserve further attention. But the effect is in any case small, and in phenomenological applications the total result, for example, in SIDIS after convolution with a fragmentation function, is strongly dominated by contributions from the valence- $x$ region and $p_{T} \lesssim 0.4 \mathrm{GeV}$, where the TMDs are sizable.

Thus, to draw an intermediate conclusion, in the valence- $x$ region the bag model strongly supports the Gaussian model with a weakly $x$-independent Gaussian widths $\left\langle p_{T}^{2}(x)\right\rangle_{\text {Gauss }}$.

Thus, the bag model results also encourage to use the so convenient Gaussian model for TMDs also in future (until the data will teach us the opposite). But how to use in practice these model predictions? Indeed, one cannot use the bag model predictions literally, since, for example, the Gaussian width of $f_{1}^{q}$ from bag model underestimates what is needed in phenomenology, see Eqs. (67) and (68).

However, the $p_{T}$-broadening mechanism [11] is in lowest order approximation polarization independent. Therefore, model predictions for ratios of widths can be expected to be more reliable and useful for practical applications. In view of the weak $x$-dependence of the $\left\langle p_{T}^{2}(x)\right\rangle_{\text {Gauss }}$ we chose $x_{v}=0.3$ as a typical valence- $x$ value and summarize the results for the $\left\langle p_{T}^{2}(x)\right\rangle_{\text {Gauss }}$, in units of the width of $f_{1}^{q}$, in Table $\square$

It is worth to compare to the results from the light-cone constituent quark model [81, 86]. In that model the wave-functions fall off with $p_{T}$ sufficiently fast, such that $\left\langle p_{T}^{2}\right\rangle$ defined in Eq. (64) exists. For the $f_{1}^{q}$ in that model $\left\langle p_{T}^{2}\right\rangle=0.080 \mathrm{GeV}^{2}$ is close to our $\left\langle p_{T}^{2}\left(x_{v}\right)\right\rangle_{\text {Gauss }}=0.077 \mathrm{GeV}^{2}$. For $g_{1}^{q}, h_{1}^{q}$ the results are comparably similar. However, in the case of $g_{1 T}^{\perp q}, h_{1 L}^{\perp q}, h_{1 T}^{\perp q}$ the light-cone constituent quark model yields smaller widths compared to the bag model, see Table [1. Twist-3 TMDs were not studied in [81, 86].

\begin{tabular}{|l|c|c|}
\hline TMD $j$ & $\begin{array}{c}\left\langle p_{T}^{2}\right\rangle \\
\text { Ref. [86] }\end{array}$ & $\begin{array}{c}\left\langle p_{T}^{2}\left(x_{v}\right)\right\rangle_{\text {Gauss }} \\
\text { (bag, here) }\end{array}$ \\
\hline$f_{1}^{q}$ & 1 & 1 \\
$g_{1}^{q}$ & 0.74 & 0.71 \\
$h_{1}^{q}$ & 0.79 & 0.85 \\
$g_{1 T}^{\perp q}, h_{1 L}^{\perp q}$ & 0.74 & 0.95 \\
$h_{1 T}^{\perp q}$ & 0.63 & 1.11 \\
$e^{q}$ & - & 0.68 \\
$g_{T}^{q}$ & - & 0.84 \\
$h_{L}^{q}$ & - & 1.01 \\
$g_{T}^{\perp q}, g_{L}^{\perp q}, h_{T}^{q}$ & - & 1.11 \\
$f^{\perp q}, h_{T}^{\perp q}$ & - & 0.94 \\
\hline
\end{tabular}

TABLE I: Average transverse momentum squares in T-even TMDs from light-cone constituent quark model [86], and the bag model (results obtained here). The $\left\langle p_{T}^{2}\right\rangle$ from [86] are defined according to [64). The bag model results for the Gaussian widths are defined according to (70) and taken at the valence- $x$ point $x_{v}=0.3$. All results are in units of the respective value for $f_{1}^{q}$, which is $\left\langle p_{T}^{2}\right\rangle=0.080 \mathrm{GeV}^{2}$ in the case of [86], and $\left\langle p_{T}^{2}\left(x_{v}\right)\right\rangle_{\mathrm{Gauss}}^{\left(f_{1}\right)}=0.077 \mathrm{GeV}^{2}$ in the case of the bag model. 


\section{WW-type approximations}

By exploring the equations of motion, twist-3 parton distributions typically can be decomposed into pieces related to leading-twist TMDs, current quark mass terms, and quark-gluon-quark correlators. The latter are often referred to as 'pure twist-3' or 'interaction dependent' terms, and are marked by a tilde. For T-even TMDs one obtains [17] (we suppress the arguments $x$ and $k_{\perp}$ )

$$
\begin{aligned}
x e^{q} & =x \tilde{e}^{q}+\frac{m_{q}}{M} f_{1}^{q}, \\
x f^{\perp q} & =x \tilde{f}^{\perp q}+f_{1}^{q}, \\
x g_{L}^{\perp q} & =x \tilde{g}_{L}^{\perp q}+g_{1}^{q}+\frac{m_{q}}{M} h_{1 L}^{\perp q}, \\
x g_{T}^{\perp q} & =x \tilde{g}_{T}^{\perp q}+g_{1 T}^{\perp q}+\frac{m_{q}}{M} h_{1 T}^{\perp q}, \\
x g_{T}^{q} & =x \tilde{g}_{T}^{q}+\frac{\vec{p}_{T}^{2}}{2 M^{2}} g_{1 T}^{\perp q}+\frac{m_{q}}{M} h_{1}^{q}, \\
x h_{L}^{q} & =x \tilde{h}_{L}^{q}-\frac{\vec{p}_{T}^{2}}{M^{2}} h_{1 L}^{\perp q}+\frac{m_{q}}{M} g_{1}^{q}, \\
x h_{T}^{q} & =x \tilde{h}_{T}^{q}-h_{1}^{q}-\frac{\vec{p}_{T}^{2}}{2 M^{2}} h_{1 T}^{\perp q}+\frac{m_{q}}{M} g_{1 T}^{\perp q}, \\
x h_{T}^{\perp q} & =x \tilde{h}_{T}^{\perp}+h_{1}^{q}-\frac{\vec{p}_{T}^{2}}{2 M^{2}} h_{1 T}^{\perp q}, \\
x g_{T}^{\prime q} & =x \tilde{g}_{T}^{\prime q}+\frac{m_{q}}{M} h_{1}^{q}-\frac{m_{q}}{M} \frac{\vec{p}_{T}^{2}}{2 M^{2}} h_{1 T}^{\perp q} .
\end{aligned}
$$

where in the last equation the notation is used $g_{T}^{\prime q} \equiv g_{T}^{q}-\frac{\vec{p}_{T}^{2}}{2 M^{2}} g_{T}^{\perp q}$ and analog for $\tilde{g}_{T}^{\prime q}$ [29]. If we systematically assume that pure twist-3 and quark mass terms are small, which we indicate symbolically and generically by $\mathcal{O}(\varepsilon)$, and integrate over transverse momenta, then we obtain the following Wandzura-Wilczek-type approximations

$$
\begin{aligned}
x e^{q}(x) & =\mathcal{O}(\varepsilon), \\
x f^{\perp q}(x) & =f_{1}^{q}(x)+\mathcal{O}(\varepsilon), \\
x g_{L}^{\perp q}(x) & =g_{1}^{q}(x)+\mathcal{O}(\varepsilon), \\
x g_{T}^{\perp q}(x) & =g_{1 T}^{\perp q}(x)+\mathcal{O}(\varepsilon), \\
x g_{T}^{q}(x) & =g_{1 T}^{\perp(1) q}(x)+\mathcal{O}(\varepsilon), \\
x h_{L}^{q}(x) & =-2 h_{1 L}^{\perp(1) q}(x)+\mathcal{O}(\varepsilon), \\
x h_{T}^{q}(x) & =-h_{1}^{q}(x)-h_{1 T}^{\perp(1)}(x)+\mathcal{O}(\varepsilon), \\
x h_{T}^{\perp q}(x) & =h_{1}^{q}(x)-h_{1 T}^{\perp(1)}(x)+\mathcal{O}(\varepsilon), \\
x g_{T}^{\prime q}(x) & =\mathcal{O}(\varepsilon) .
\end{aligned}
$$

What these approximations have in common with the classic Wandzura-Wilczek (WW) approximation is that pure twist-3 and current quark mass terms are neglected. However, the neglected operators are different in all cases.

Figs. 66 -6. compare respectively the left- (solid lines) and right-hand-sides (dashed lines) of the Eqs. (80 88), assuming that $\mathcal{O}(\varepsilon)$ is zero in each case. In all Figures the same scale is used in order to better compare the magnitudes of the various functions. First we observe that $x e^{q}(x)$ and $x g_{T}^{\prime q}(x)$ are not zero, as one would expect on the basis on the WW-type approximations, though the functions are not large, see Figs. [6a and 6].

Next we remark that the WW-type approximations for $x f^{\perp q}(x), x h_{L}^{q}(x), x h_{T}^{\perp q}(x)$ can be considered as roughly supported by the bag model, see Figs. 6b, 6f, 6h. In the remaining cases, however, the WW-type approximations

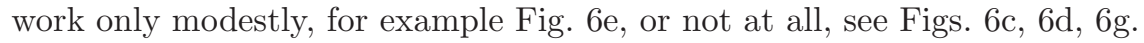

At first glance one might be surprised that the WW-type approximations are not exactly fulfilled in a no-gluon model with massless quarks, as apparently there are no contributions from quark-gluon and mass terms. However, here one has to recall that the WW-type approximations originate from applying $Q C D$ equations of motion, and 
separating leading and subleading twist terms. In principle, one could repeat this game in the bag model, too. As the quarks are not free (but confined by the bag), one would consequently encounter certain "interaction dependent" tildeterms in the model, too. These bag-model tilde-terms can be seen directly in the case of $e^{q}(x), g_{T}^{\prime q}(x)$ in Figs. 6r, 66. In the other plots in Fig. 6 they are apparent as the differences between the solid and dashed lines. It has been argued that the bag, which is a model for confinement, in some sense mimics the effects of gluons [103]. However, to which extent the bag-model interaction-dependent terms are able to estimate reliably the QCD interaction-dependent terms, remains to be seen.

In any case, it is interesting to observe that the bag model roughly supports the WW-type approximation for $x f^{\perp u}(x)$, see Fig. 6b, which played an important role in the interpretation [59] of the EMC data on the azimuthal asymmetry $A_{U U}^{\cos \phi}$ in unpolarized SIDIS [36] as being due to the Cahn effect [8].
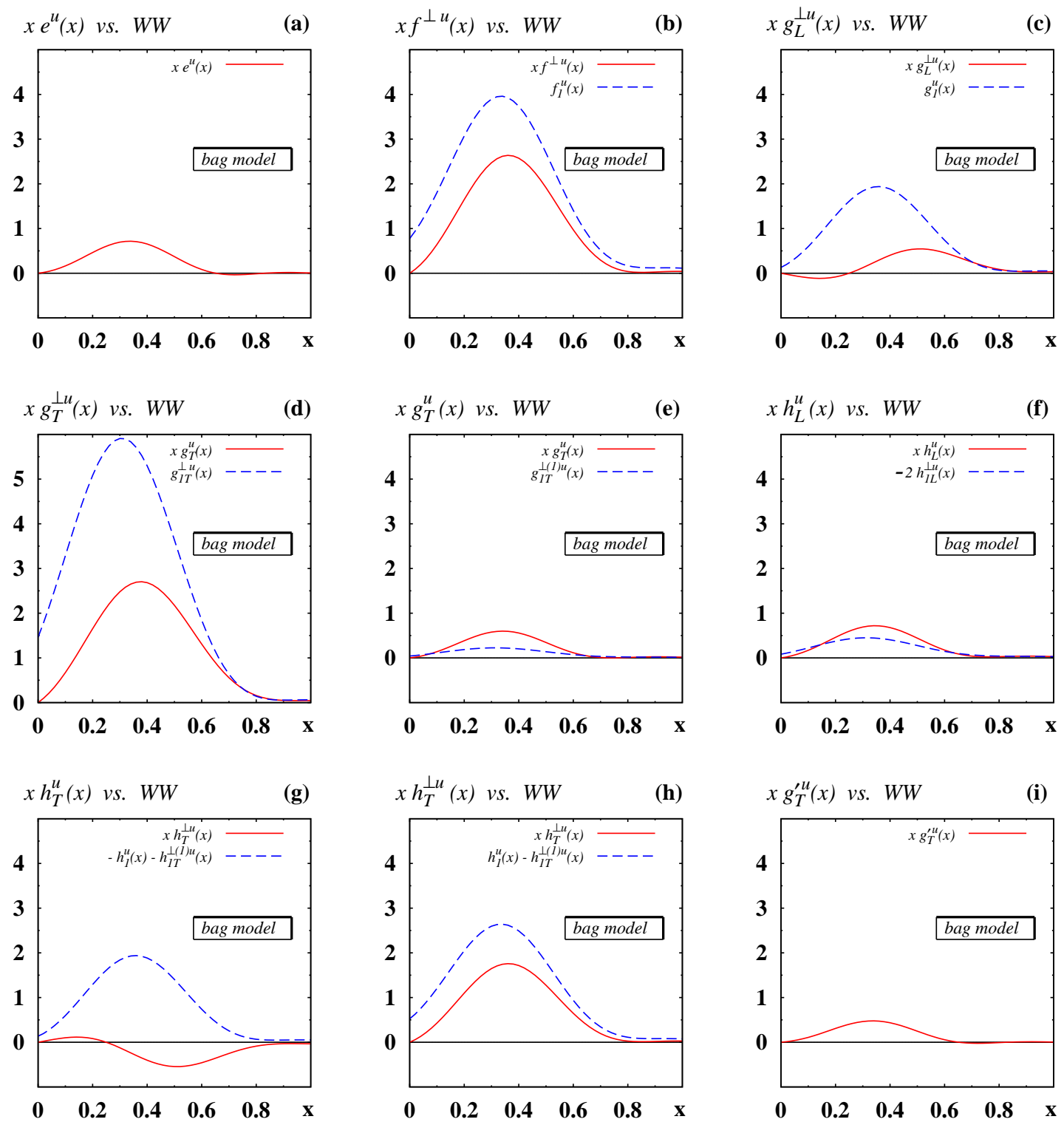

FIG. 6: Test of WW-type approximations in the bag model at the low scale. The solid lines show $x$ times twist-3 TMDs, as given on the left-hand-sides on the Eqs. (80 88). The dashed lines are twist-2 TMDs (or their transverse moments or linear combinations thereof) as given on the right-hand-sides of the Eqs. (80, 88). If the tilde- and mass-terms defined in terms of the QCD equations of motion vanished, the solid and dashed lines would coincide, see text. In Figs. (a) and (i) no dashed lines appear because the right-hand-sides of the Eqs. (80) and (88) are in the WW-type approximation. 
Having discussed the WW-type approximations for TMDs, whose usefulness remains to be tested [99], it is interesting to have a look back on the "classic WW-approximations" for $g_{T}^{q}(x)$ and $h_{L}^{q}(x)$ [103, 109], which are distinguished from the WW-type approximations in that in their derivations the notion and complications of transverse parton momenta does not need to be involved [103, 109], though can be considered [101, 124]. These are therefore in a certain sense "collinear" approximations. They are given by

$$
\begin{aligned}
& g_{T}^{q}(x) \stackrel{\mathrm{WW}}{\approx} \int_{x}^{1} \frac{\mathrm{d} y}{y} g_{1}^{a}(y), \\
& h_{L}^{q}(x) \stackrel{\mathrm{WW}}{\approx} 2 x \int_{x}^{1} \frac{\mathrm{d} y}{y^{2}} h_{1}^{a}(y) .
\end{aligned}
$$

Figs. $7 \mathrm{f}$ and $7 \mathrm{~b}$ show to which extent the WW approximations are supported by the bag model: moderately in the case of $g_{T}^{q}(x)$, and somewhat better in the case of $h_{L}^{q}(x)$. (In Figs. $7 \mathrm{a}$ and $7 \mathrm{~b}$ we compare $x$ times the functions and their WW-approximations, because at small $x \lesssim 0.1$ the approximations (89, 90) are poorly supported, which should not worry us as the bag model is expected to be more meaningful in the valence- $x$ region, see Sec. [II])

Finally, we remark that by combining the classic WW approximations for $g_{T}^{q}(x)$ in Eq. [89) [109], and $h_{L}^{q}(x)$ in Eq. (90) [103], with respectively the WW-type approximations in Eqs. (84) and (85), one obtains in principle a further class of approximations [99], which we shall denote by "WW \& type" (short for WW and WW-type) approximations. These approximations relate leading twist TMDs to twist-2 parton distributions as follows [98, 99]

$$
\begin{aligned}
& g_{1 T}^{\perp(1) a}(x) \stackrel{\text { WW } \& \text { type }}{\approx} \quad x \int_{x}^{1} \frac{\mathrm{d} y}{y} g_{1}^{a}(y), \\
& h_{1 L}^{\perp(1) a}(x) \stackrel{\text { WW } \underset{\approx}{\approx} \text { type }}{\approx}-x^{2} \int_{x}^{1} \frac{\mathrm{d} y}{y^{2}} h_{1}^{a}(y) .
\end{aligned}
$$

These approximations were used in literature in order to make estimates for certain double [97] and single [98] spin asymmetries in SIDIS. In Figs. $7 \mathrm{r}$ and $7 \mathrm{~d}$ we test the quality of these approximations in the bag model at the low scale. In both cases we observe that the approximations tend to overestimate the magnitude of the true model results for $g_{1 T}^{\perp(1) a}(x)$ and $h_{1 L}^{\perp(1) a}(x)$, somewhat more in the former case and less in the latter case.

It is, however, difficult to suspect on the basis of these observations at the low scale of the bag model, whether the predictions from Refs. [97, 98] will similarly overestimate data. The evolution effects may play an important role.
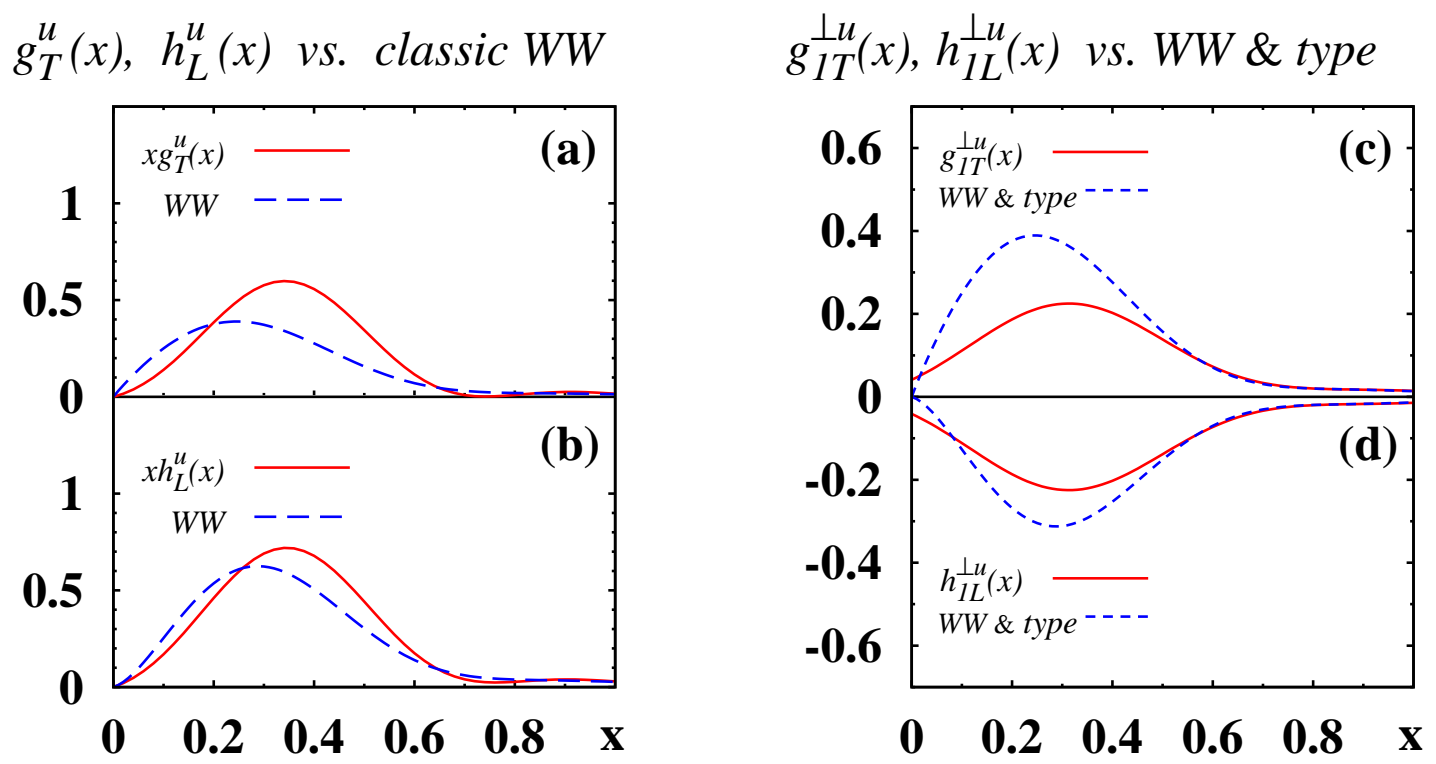

FIG. 7: (a) and (b): The test of the classic WW approximations for $g_{T}^{q}(x)$ [109] and $h_{L}^{q}(x)$ [103], see Eqs. (89, 90), in bag model. (c) and (d): Test of the approximations for $g_{1 T}^{\perp(1) q}(x)$ and $h_{1 L}^{\perp(1) q}(x)$ in Eqs. (91, 92), which result from combining Eqs. (84 85) and (89, 90). All results refer to the low bag model scale. 
Though the precise evolution pattern of polarized transverse momentum dependent distribution functions is not yet fully understood, it seems reasonable to expect the evolution from a low hadronic to experimentally relevant scales will "shift" the $x$-shape of the TMDs towards smaller $x$, see also [25]. In other words, the region of valence- and large- $x$ in the bag model at its low scale, say $0.3 \lesssim x \lesssim 0.8$, could be what will be just relevant at higher scales in the experiments at COMPASS, Jefferson Lab or HERMES. Remarkably, in the region of larger $x \gtrsim 0.3$ the approximations (91, 92) work reasonably well, see Figs. 7; and 7d.

\section{Orbital angular momentum}

For completeness we include here also the bag model results for the nucleon spin decomposition. Of course, it is well known that in the bag model $65 \%$ of the nucleon spin is due to the intrinsic spin of the quarks, and the remaining $35 \%$ are due to quark orbital motion 104], see also the study of this issue in the bag model in the context of generalized parton distribution functions [125]. However, what is new here is that we obtain this information from the pretzelosity distribution, namely

$$
\begin{array}{rlrl}
2 L_{u}^{3} & \equiv-2 \int \mathrm{d} x h_{1 T}^{\perp(1) u}(x)=0.46, & 2 L_{d}^{3} \equiv-2 \int \mathrm{d} x h_{1 T}^{\perp(1) d}(x)=-0.11, & 2 L_{Q}^{3}=2 L_{u}^{3}+2 L_{d}^{3}=0.35, \\
2 S_{u}^{3} \equiv \int \mathrm{d} x g_{1}^{u}(x)=0.87, & 2 S_{d}^{3} \equiv \int \mathrm{d} x g_{1}^{d}(x)=-0.22, & 2 S_{Q}^{3}=2 S_{u}^{3}+2 S_{d}^{3}=0.65, \\
2 J_{u}^{3}=2 L_{u}^{3}+2 S_{u}^{3}=\frac{4}{3}, & 2 J_{d}^{3}=2 L_{d}^{3}+2 S_{d}^{3}=-\frac{1}{3}, & 2 J_{Q}^{3}=2 L_{Q}^{3}+2 S_{Q}^{3}=1 .
\end{array}
$$

This is a typical result for relativistic quark models at low hadronic scales [126, 127]. We remark that the MIT bag also reasonably well describes the axial coupling constant: the Bjorken sum rule yields $g_{A}=\int \mathrm{d} x\left(g_{1}^{u}-g_{1}^{d}\right)(x)=1.09$ vs. 1.26 in experiment. (At this point it is interesting to note that experimentally the Bjorken sum rule is verified with not much better accuracy than that [128], see also [129] for a recent comparison of QCD and data.)

However, in order to compare such numbers to phenomenology or lattice QCD [130] one needs to carefully take into account evolution effects [131] which, in the case of bag model, is supposed to start at a very low hadronic scale [132]. Evolution techniques possibly suitable for that were discussed in the context of the Bjorken sum rule in [129]. 


\section{CONCLUSIONS}

We presented a study of leading- and subleading-twist TMDs in the MIT bag model. Since this model lacks explicit gluon degrees of freedom, the Wilson-link needed in QCD to render the definitions of TMDs color gauge invariant is absent. As a consequence T-odd TMDs vanish. Attempts to model T-odd TMDs in the bag were presented in 91 93. In this work we have focused on the 14 T-even TMDs (6 leading- and 8 subleading-twist).

Another consequence of the absence of the Wilson-link (in any effective approach with global color symmetry only), is that certain relations hold among TMDs, the so-called LIRs [17, 100]. There are 5 such LIRs among the 14 T-even leading- and subleading-twist TMDs, and we have proven that they are all satisfied in the bag model.

Recently further relations among TMDs were found in models. One of the motivations of this work was therefore to shed some light on how such quark model relations arise. We have shown that in the MIT bag model there are not more and not less than 9 linear and 2 non-linear relations among the 14 T-even leading- and subleading-twist TMDs. We reviewed in detail that some of these relations are supported also in other quark models [79, 81, 82, 85] .

One of those linear relations, found in our previous bag model study [80], connects the difference of $g_{1}^{q}$ and $h_{1}^{q}$ to the (1)-moment of pretzelosity. It was confirmed in several other [81, 82, 85] though not all [85] quark models. What makes this relation particularly interesting, is the observation [84] that such a difference between helicity and transversity distributions is related in a light-cone SU(6) quark-diquark model [133] to quark orbital angular momentum (OAM).

Although intuitively the idea of quark orbital motion is associated with TMDs, this is to best of our knowledge the first 'rigorous' connection of a TMD to OAM - in a model, of course. While in gauge theories there is no gaugeinvariant definition of an OAM field operator, in quark models the situation is simpler and there are no ambiguities. Another important motivation for our study was therefore to investigate whether there exists any connection between pretzelosity and quark OAM in the bag model. The answer is yes, agrees with the findings of [84], and reads

$$
L_{q}^{3}=(-1) \int \mathrm{d} x h_{1 T}^{\perp(1) q}(x) .
$$

Thus, by measuring the single spin asymmetry $A_{U T}^{\sin \left(3 \phi-\phi_{S}\right)}$ due to $h_{1 T}^{\perp q}$ [17] one could access information on OAM. Of course, the relation (96) is model-dependent. But it is supported by two independent approaches, bag model (here) and light-cone SU(6) quark-diquark model [84]. Moreover, at least in the context of bag model, the information on OAM gained from pretzelosity, Eq. (96), is equivalent to what one can learn from generalized parton distribution functions [125]. It will be exciting to see to what extent experimental information on TMDs and generalized parton distribution will, on the basis of a quark model interpretation, converge to give a compatible picture of OAM.

The pretzelosity distribution $h_{1 T}^{\perp q}$ seems to play in this context a central role. It is also related to the non-sphericity of the spin-distribution in the nucleon [32]. It is interesting to ask, whether a quark model relation of the type (96) may inspire a way to establish a rigorous connection between TMDs and OAM in QCD. The task is demanding, as we observe (96) on the level of matrix elements only. Further studies in effective approaches, and numerical results from lattice QCD could provide valuable insights. For first attempts to study TMDs on a lattice see [34].

The third main result of this work concerns practical aspects which are of interest for phenomenology. For example, in many phenomenological studies it is assumed that in TMDs the $x$ - and $p_{T}$-dependencies factorize, and the latter is "Gaussian". Many authors have stressed that in their models the $x$ - and $p_{T}$-dependencies of TMDs are non-factorizing and non-Gaussian. But in practical applications the Gaussian Ansatz works with a useful accuracy, e.g. [58 60].

How to reconcile these observations? In order to address this question, we introduced the notion of a (in general $x$-dependent) 'Gaussian width' which can be applied to any TMD. This effective Gaussian width is designed such that it reproduces the $p_{T}$-dependence of the TMD exactly in a vicinity of $p_{T}=0$ by definition. Although in the bag we also observe non-factorizing, non-Gaussian $x$ - and $p_{T}$-dependencies, in this way we made two interesting observations. In the valence- $x$ region (and we speak here about a low hadronic scale), this effective 'Gaussian width' turns out to be only weakly $x$-dependent. Moreover, such an effective Gaussian Ansatz approximates reasonably well the exact model results not only in the vicinity of $p_{T}=0$, but up to $p_{T} \lesssim \mathcal{O}\left(M_{N}\right)$.

This is good news for phenomenological studies for two reasons: azimuthal asymmetries in Drell-Yan or SIDIS are dominated by intrinsic transverse parton momenta [8 10], and one expects azimuthal and (single) spin phenomena to be sizable in the valence- $x$ region. And this is where we find the Gaussian Ansatz to be a useful approximation. Surely, care is required when sea-quark effects start to play a role, and for a precision treatment of transverse momenta one has to use a rigorous approach such as the Collins-Soper-Sterman formalism [11] as implemented in [134].

Finally, we used the model results to test the Wandzura-Wilczek-type approximations [97 100] which were suggested as, at the presently early stage, useful tools for first interpretations of data. These approximations consist in neglect 'pure-interaction-dependent' terms (in QCD: quark-gluon correlations, in the bag model: bag boundary conditions). We observe that, for some TMDs these are fair approximations in the valence- $x$ region.

To conclude, though obtained in a simple model, our results bear many interesting insights, and we hope they will stimulate further studies in quark models. 
Acknowledgements. A. E. is supported by the Grants RFBR 09-02-01149 and 07-02-91557, RF MSE RNP 2.1.1/2512 (MIREA) and by the Heisenberg-Landau Program of JINR. The work was supported in part by DOE contract DE-AC05-06OR23177, under which Jefferson Science Associates, LLC, operates the Jefferson Lab. F. Y. is grateful to RIKEN, Brookhaven National Laboratory and the U.S. Department of Energy (contract number DEAC02-98CH10886) for providing the facilities essential for the completion of this work.

\section{Appendix A: Proofs of LIRs}

In this Appendix we prove the LIRs (34) 38). Thanks to the model relations discussed in Sec. IIIB, we do not need to prove every LIR. For example, if we prove the LIR (34) then also the LIR (35) holds due to the relations (42, 45, 46), which can be seen conveniently by combining (45, 46) to form (53). Similarly, if we prove the LIR (36) then it is clear, that also the LIR (37) holds, due to the model relations (43) 44). Finally, (38) is evidently true, c.f. the model relation (47). Thus, it is sufficient to demonstrate, for example, that the LIRs (34) and (36) hold.

In order to prove (36) we rewrite the expression for $h_{1 T}^{\perp(1)}(x)$ in a convenient for our purposes way. Recalling that $k$ is a function of $k_{\perp}$ and $k_{z}=x M_{N}-\omega / R_{0}$ according to (18), we may write

$$
h_{1 T}^{\perp(1) q}(x)=P_{q} A \int \mathrm{d}^{2} k_{\perp} \frac{k_{\perp}^{2}}{2 M_{N}^{2}}\left[-2 \widehat{M}_{N}^{2} t_{1}^{2}(k)\right]=P_{q} A \int \mathrm{d}^{3} q \int \frac{\mathrm{d} \tau}{2 \pi} e^{i\left(k_{z}-q \cos \theta\right) \tau}\left[-\sin ^{2} \theta t_{1}^{2}(q)\right]
$$

where we replaced the transverse momentum integral by an integration over the independent variables $\vec{q}$ with $q=|\vec{q}|$, using a delta-function $\int \mathrm{d} \tau /(2 \pi) e^{i\left(k_{z}-q_{z}\right) \tau}=\delta\left(q_{z}-k_{z}\right)$, and the spherical coordinates $q_{z}=q \cos \theta$ and $q_{\perp}^{2}=q^{2} \sin { }^{2} \theta$. Next we differentiate (A1) with respect to $x$, recalling that the $x$-dependence is 'hidden' only in $k_{z}$ according to (18) with $\frac{\mathrm{d}}{\mathrm{d} x} k_{z}=M_{N}$. We obtain

$$
\begin{aligned}
\frac{\mathrm{d}}{\mathrm{d} x} h_{1 T}^{\perp(1) q}(x) & =P_{q} A \int \mathrm{d}^{3} q \int \frac{\mathrm{d} \tau}{2 \pi} e^{i\left(k_{z}-q \cos \theta\right) \tau}\left(i M_{N} \tau\right)\left[-\sin ^{2} \theta t_{1}^{2}(q)\right] \\
& =P_{q} A \int \mathrm{d}^{3} q \int \frac{\mathrm{d} \tau}{2 \pi}\left\{-\frac{M_{N}}{q} \frac{\mathrm{d}}{\mathrm{d} \cos \theta} e^{i\left(k_{z}-q \cos \theta\right) \tau}\right\}\left[-\sin ^{2} \theta t_{1}^{2}(q)\right] \\
& =P_{q} A \int \mathrm{d}^{3} q \int \frac{\mathrm{d} \tau}{2 \pi} \frac{M_{N}}{q} e^{i\left(k_{z}-q \cos \theta\right) \tau}\left[-2 \cos \theta t_{1}^{2}(q)\right] \\
& =P_{q} A \int \mathrm{d}^{3} q \delta\left(q_{z}-k_{z}\right)\left[2 M_{N} q_{z} \frac{t_{1}^{2}(q)}{q^{2}}\right] \\
& =P_{q} A \int \mathrm{d}^{2} k_{\perp}\left[2 \widetilde{M}_{N} \widetilde{k}_{z} t_{1}^{2}(k)\right] \\
& \equiv-h_{T}^{q}(x)
\end{aligned}
$$

where we interchanged the order of the integrations and differentiation, which is legitimate in our case, integrated by parts in the third step, and finally recovered the expression for $(-1) h_{T}^{q}(x)$, which completes the proof of the LIR (36). The proof of the LIR (34) is straightforward, and consists of repeating steps analog to (A1) A2).

It is interesting to remark that a different version of the LIR (36) is the following

$$
h_{T}^{(1) q}(x) \stackrel{\text { LIR }}{=}-\frac{1}{2} \frac{\mathrm{d}}{\mathrm{d} x} h_{1 T}^{\perp(2) q}(x),
$$

with the subtlety that the (2)-moment of pretzelosity is divergent, but its derivative with respect to $x$ is finite, i.e. the same careful treatment is required as in the case of the (1)-moment of $f_{1}^{q}$ discussed in detail in Sec. VB. The numerical bag model results for TMDs satisfy all LIRs including the version (A3). 


\section{Appendix B: Proofs of inequalities}

This Appendix contains the explicit demonstrations that the quark TMDs from the bag model satisfy the inequalities (54 58). We recall that, in the present version of the model, the T-odd TMDs $f_{1 T}^{\perp q}$ and $h_{1}^{\perp q}$ are absent and that the inequalities for antiquarks are violated, see Sec. If for a detailed discussion.

In order to check the inequalities in (54) we work directly with the model expressions. With $\widehat{k}_{z}=k_{z} / \sqrt{k_{\perp}^{2}+k_{z}^{2}}$ we have $-1<\widehat{k}_{z}<1$ which we use below in (B1), $-1 \leq\left(2 \widehat{k}_{z}^{2}-1\right) \leq 1$ we use in (B2), and $\widehat{k}_{z}^{2}<1$ used in (B3),

$$
\begin{aligned}
& f_{1}^{q}\left(x, k_{\perp}\right)=N_{q} A\left[t_{0}^{2}+2 \widehat{k}_{z} t_{0} t_{1}+t_{1}^{2}\right] \geq N_{q} A\left(t_{0}-t_{1}\right)^{2} \geq 0, \\
& g_{1}^{q}\left(x, k_{\perp}\right)=P_{q} A\left[t_{0}^{2}+2 \widehat{k}_{z} t_{0} t_{1}+\left(2 \widehat{k}_{z}^{2}-1\right) t_{1}^{2}\right] \leq P_{q} A\left[t_{0}^{2}+2 \widehat{k}_{z} t_{0} t_{1}+t_{1}^{2}\right]=\frac{P_{q}}{N_{q}} f_{1}^{q}\left(x, k_{\perp}\right), \\
& h_{1}^{q}\left(x, k_{\perp}\right)=P_{q} A\left[t_{0}^{2}+2 \widehat{k}_{z} t_{0} t_{1}+\widehat{k}_{z}^{2} t_{1}^{2}\right] \leq P_{q} A\left[t_{0}^{2}+2 \widehat{k}_{z} t_{0} t_{1}+t_{1}^{2}\right]=\frac{P_{q}}{N_{q}} f_{1}^{q}\left(x, k_{\perp}\right),
\end{aligned}
$$

The inequalities (B2, B33) imply $\left|g_{1}^{q}\left(x, k_{\perp}\right)\right|<f_{1}^{q}\left(x, k_{\perp}\right)$ and $\left|h_{1}^{q}\left(x, k_{\perp}\right)\right|<f_{1}^{q}\left(x, k_{\perp}\right)$, because for the nucleon in SU(6)

$$
\left|\frac{P_{q}}{N_{q}}\right|<1, q=u, d
$$

In order to check the inequalities (55, 56) it is convenient to explore the model relations (39) 477). For example, because of (B4) the relation (39) immediately implies that for the nucleon the Soffer inequality (55) is a true inequality $\left|h_{1}^{q}\left(x, k_{\perp}\right)\right|<\frac{1}{2}\left(f_{1}^{q}\left(x, k_{\perp}\right)+g_{1}^{q}\left(x, k_{\perp}\right)\right)$, see also [16]. Similarly, by using the relations [39 [55) to eliminate transversity, we conclude with (B4) that (56) is a true inequality, i.e. $\left|h_{1 T}^{\perp q}\left(x, k_{\perp}\right)\right|<\frac{1}{2}\left(f_{1}^{q}\left(x, k_{\perp}\right)-g_{1}^{q}\left(x, k_{\perp}\right)\right)$.

In order to verify the inequalities (57, 58) we explore the linear (39, 47) and non-linear (51, 52) model relations. It is sufficient, thanks to the absence of the T-odd TMDs $f_{1 T}^{\perp q}$ and $h_{1}^{\perp q}$ and the relation (42), to prove one of these inequalities, let us say (58). For that we multiply (51) by $\left(k_{\perp}^{2} / 2 M_{N}^{2}\right)^{2}$, and eliminate transversity by means of the relation (39) and $h_{1 T}^{\perp(1) q}\left(x, k_{\perp}\right)$ by means of the relations (39] 45). This yields

$$
h_{1 L}^{\perp(1) q}\left(x, k_{\perp}\right)^{2}=\frac{k_{\perp}^{2}}{4 M_{N}^{2}}\left(\frac{P_{q}^{2}}{N_{q}^{2}} f_{1}^{q}\left(x, k_{\perp}\right)^{2}-g_{1}^{q}\left(x, k_{\perp}\right)^{2}\right)<\frac{k_{\perp}^{2}}{4 M_{N}^{2}}\left(f_{1}^{q}\left(x, k_{\perp}\right)^{2}-g_{1}^{q}\left(x, k_{\perp}\right)^{2}\right),
$$

where in the last step we explored (B4).

It is interesting to remark that, except for $f_{1}^{q}\left(x, k_{\perp}\right) \geq 0$, all the other inequalities in (54 58) are true inequalities for the proton in $\mathrm{SU}(6)$, i.e. they are never saturated. For other baryons in $\mathrm{SU}(6)$ saturations may occur, for example, for strange quarks in the $\Lambda$ hyperon, where $N_{s}=P_{s}=1$, see [116] for a related discussion.

[1] J. C. Collins, Acta Phys. Polon. B 34 (2003) 3103.

[2] J. C. Collins, T. C. Rogers and A. M. Stasto, Phys. Rev. D 77 (2008) 085009.

[3] J. C. Collins, F. Hautmann, Phys. Lett. B 472 (2000) 129, JHEP 0103 (2001) 016.

[4] F. Hautmann, Phys. Lett. B 655 (2007) 26.

[5] J. C. Collins and D. E. Soper, Nucl. Phys. B 193, 381 (1981) [Erratum-ibid. B 213, 545 (1983)].

[6] X. D. Ji, J. P. Ma and F. Yuan, Phys. Rev. D 71, 034005 (2005) arXiv:hep-ph/0404183, Phys. Lett. B 597, 299 (2004) arXiv:hep-ph/0405085.

[7] J. C. Collins and A. Metz, Phys. Rev. Lett. 93, 252001 (2004) arXiv:hep-ph/0408249.

[8] R. N. Cahn, Phys. Lett. B 78 (1978) 269.

[9] A. König and P. Kroll, Z. Phys. C 16 (1982) 89.

[10] P. Chiappetta and M. Le Bellac, Z. Phys. C 32 (1986) 521.

[11] J. C. Collins, D. E. Soper and G. Sterman, Nucl. Phys. B 250, 199 (1985).

[12] D. W. Sivers, Phys. Rev. D 41, 83 (1990), Phys. Rev. D 43, 261 (1991).

[13] A. V. Efremov, L. Mankiewicz and N. A. Tornqvist, Phys. Lett. B 284 (1992) 394.

[14] J. C. Collins, Nucl. Phys. B 396, 161 (1993) arXiv:hep-ph/9208213.

[15] J. C. Collins, S. F. Heppelmann and G. A. Ladinsky, Nucl. Phys. B 420 (1994) 565 arXiv:hep-ph/9305309.

[16] A. Kotzinian, Nucl. Phys. B 441 (1995) 234 arXiv:hep-ph/9412283.

[17] P. J. Mulders and R. D. Tangerman, Nucl. Phys. B 461 (1996) 197 and 484 (1997) 538E arXiv:hep-ph/9510301. 
[18] D. Boer and P. J. Mulders, Phys. Rev. D 57, 5780 (1998) arXiv:hep-ph/9711485].

[19] D. Boer, R. Jakob and P. J. Mulders, Nucl. Phys. B 504 (1997) 345 arXiv:hep-ph/9702281].

[20] D. Boer, Phys. Rev. D 60, 014012 (1999) arXiv:hep-ph/9902255.

[21] A. Bacchetta, M. Boglione, A. Henneman and P. J. Mulders, Phys. Rev. Lett. 85, 712 (2000) arXiv:hep-ph/9912490.

[22] S. J. Brodsky, D. S. Hwang and I. Schmidt, Phys. Lett. B 530, 99 (2002) arXiv:hep-ph/0201296; Nucl. Phys. B 642, 344 (2002) arXiv:hep-ph/0206259.

[23] J. C. Collins, Phys. Lett. B 536, 43 (2002) arXiv:hep-ph/0204004.

[24] A. V. Belitsky, X. Ji and F. Yuan, Nucl. Phys. B 656, 165 (2003) arXiv:hep-ph/0208038. X. D. Ji and F. Yuan, Phys. Lett. B 543, 66 (2002) arXiv:hep-ph/0206057.

D. Boer, P. J. Mulders and F. Pijlman, Nucl. Phys. B 667, 201 (2003) arXiv:hep-ph/0303034.

[25] I. O. Cherednikov and N. G. Stefanis, Phys. Rev. D 77, 094001 (2008) arXiv:0710.1955 [hep-ph]]. Nucl. Phys. B 802, 146 (2008) arXiv:0802.2821 [hep-ph]]. Phys. Rev. D 80, 054008 (2009) arXiv:0904.2727 [hep-ph]]; Mod. Phys. Lett. A 24, 2913 (2009) arXiv:0910.3108 [hep-ph]].

[26] M. Burkardt, Phys. Rev. D 66, 114005 (2002) arXiv:hep-ph/0209179; Phys. Rev. D 69, 057501 (2004) arXiv:hep-ph/0311013; arXiv:0709.2966 [hep-ph].

[27] P. V. Pobylitsa, arXiv:hep-ph/0301236.

[28] K. Goeke, A. Metz and M. Schlegel, Phys. Lett. B 618, 90 (2005) arXiv:hep-ph/0504130.

[29] A. Bacchetta, M. Diehl, K. Goeke, A. Metz, P. J. Mulders and M. Schlegel, JHEP 0702 (2007) 093 arXiv:hep-ph/0611265.

[30] S. J. Brodsky and F. Yuan, Phys. Rev. D 74 (2006) 094018 arXiv:hep-ph/0610236.

[31] H. Avakian, S. J. Brodsky, A. Deur and F. Yuan, Phys. Rev. Lett. 99, 082001 (2007) arXiv:0705.1553 [hep-ph]].

[32] G. A. Miller, Phys. Rev. C 76, 065209 (2007) arXiv:0708.2297 [nucl-th]].

[33] S. Arnold, A. Metz and M. Schlegel, Phys. Rev. D 79 (2009) 034005.

[34] Ph. Hägler, B. U. Musch, J. W. Negele, A. Schäfer, Europhys. Lett. 88, 61001 (2009) [arXiv:0908.1283v1 [hep-lat]]. B. U. Musch, P. Hägler, A. Schäfer, D. B. Renner, J. W. Negele [LHPC Collaboration], PoS LC2008, 053 (2008) arXiv:0811.1536 [hep-lat]].

[35] S. J. Brodsky, B. Pasquini, B. Xiao and F. Yuan, arXiv:1001.1163 [hep-ph].

[36] M. Arneodo et al. [European Muon Collaboration], Z. Phys. C 34 (1987) 277.

[37] M. Osipenko et al. [CLAS Collaboration], Phys. Rev. D 80 (2009) 032004.

[38] F. Giordano, R. Lamb [HERMES Collaboration], AIP Conf. Proc. 1149 (2009) 423. A. Bressan et al. [COMPASS Collaboration], arXiv:0907.5511 [hep-ex].

[39] A. Airapetian et al. [HERMES Collaboration], Phys. Rev. Lett. 84, 4047 (2000) arXiv:hep-ex/9910062], Phys. Rev. D 64, 097101 (2001) arXiv:hep-ex/0104005, Phys. Lett. B 562, 182 (2003) arXiv:hep-ex/0212039.

[40] H. Avakian et al. [CLAS Collaboration], Phys. Rev. D 69, 112004 (2004) arXiv:hep-ex/0301005. H. Avakian, P. Bosted, V. Burkert and L. Elouadrhiri [CLAS], AIP Conf. Proc. 792 (2005) 945 arXiv:nucl-ex/0509032.

[41] A. Airapetian et al. [HERMES Collaboration], Phys. Lett. B 622, 14 (2005) arXiv:hep-ex/0505042, Phys. Lett. B 648 (2007) 164 arXiv:hep-ex/0612059.

[42] W. Gohn, H. Avakian, K. Joo, and M. Ungaro, AIP Conf. Proc. 1149 (2009) 461.

[43] A. Airapetian et al. [HERMES Collaboration], Phys. Rev. Lett. 94, 012002 (2005) arXiv:hep-ex/0408013.

[44] V. Y. Alexakhin et al. [COMPASS Collaboration], Phys. Rev. Lett. 94, 202002 (2005) arXiv:hep-ex/0503002].

[45] M. Diefenthaler, AIP Conf. Proc. 792 (2005) 933, arXiv:0706.2242 [hep-ex], arXiv:hep-ex/0612010

L. L. Pappalardo [HERMES Collaboration], Eur. Phys. J. A 38, 145 (2008).

[46] I. M. Gregor [HERMES Collaboration], Acta Phys. Polon. B 36, 209 (2005).

[47] E. S. Ageev et al. [COMPASS Collaboration], Nucl. Phys. B 765 (2007) 31 arXiv:hep-ex/0610068].

[48] A. Airapetian et al. [HERMES Collaboration], JHEP 0806, 017 (2008) arXiv:0803.2367 [hep-ex]], Phys. Rev. Lett. 103, 152002 (2009) arXiv:0906.3918 [hep-ex]].

[49] A. Kotzinian [on behalf of the COMPASS collaboration], arXiv:0705.2402 [hep-ex].

[50] A. Airapetian et al. [HERMES Collaboration], arXiv:0906.2478 [hep-ex].

[51] S. Falciano et al. [NA10 Collaboration], Z. Phys. C 31 (1986) 513.

M. Guanziroli et al. [NA10 Collaboration], Z. Phys. C 37 (1988) 545.

[52] J. S. Conway et al., Phys. Rev. D 39 (1989) 92.

[53] L. Y. Zhu et al., Phys. Rev. Lett. 99 (2007) 082301, 102 (2009) 182001.

[54] K. Abe et al. [Belle Collaboration], Phys. Rev. Lett. 96, 232002 (2006) arXiv:hep-ex/0507063.

[55] A. Ogawa, M. Grosse-Perdekamp, R. Seidl and K. Hasuko, arXiv:hep-ex/0607014

[56] R. Seidl, M. Grosse-Perdekamp and A. Ogawa [Belle Collaboration], |arXiv:0805.2975] [hep-ex].

[57] A. Vossen, R. Seidl, M. Grosse-Perdekamp, M. Leitgab, A. Ogawa and K. Boyle, arXiv:0912.0353 [hep-ex].

[58] U. D'Alesio and F. Murgia, Phys. Rev. D 70, 074009 (2004) arXiv:hep-ph/0408092.

[59] M. Anselmino, M. Boglione, U. D'Alesio, A. Kotzinian, F. Murgia and A. Prokudin, Phys. Rev. D 71 (2005) 074006 arXiv:hep-ph/0501196.

[60] J. C. Collins, A. V. Efremov, K. Goeke, S. Menzel, A. Metz and P. Schweitzer, Phys. Rev. D 73 (2006) 014021. J. C. Collins et al., Phys. Rev. D 73, 094023 (2006) arXiv:hep-ph/0511272.

[61] A. V. Efremov, K. Goeke, S. Menzel, A. Metz and P. Schweitzer, Phys. Lett. B 612, 233 (2005) arXiv:hep-ph/0412353.

[62] W. Vogelsang and F. Yuan, Phys. Rev. D 72 (2005) 054028 arXiv:hep-ph/0507266.

[63] A. V. Efremov, K. Goeke and P. Schweitzer, Phys. Rev. D 73, 094025 (2006) arXiv:hep-ph/0603054.

[64] M. Anselmino, M. Boglione, U. D'Alesio, A. Kotzinian, F. Murgia, A. Prokudin and C. Turk, Phys. Rev. D 75, 054032 
(2007) arXiv:hep-ph/0701006.

[65] S. Arnold et al., arXiv:0805.2137 [hep-ph].

[66] M. Anselmino et al., Eur. Phys. J. A 39 (2009) 89 arXiv:0805.2677 [hep-ph].

[67] V. Barone, Z. Lu and B. Q. Ma, Phys. Lett. B 632, 277 (2006) arXiv:hep-ph/0512145].

V. Barone, A. Prokudin and B. Q. Ma, Phys. Rev. D 78, 045022 (2008) arXiv:0804.3024 [hep-ph]].

V. Barone, S. Melis and A. Prokudin, arXiv:0912.5194 [hep-ph].

[68] E. De Sanctis, W. D. Nowak and K. A. Oganessian, Phys. Lett. B 483, 69 (2000) arXiv:hep-ph/0002091.

K. A. Oganessian, N. Bianchi, E. De Sanctis and W. D. Nowak, Nucl. Phys. A 689, 784 (2001) arXiv:hep-ph/0010261].

[69] M. Anselmino and F. Murgia, Phys. Lett. B 483 (2000) 74 arXiv:hep-ph/0002120.

[70] A. V. Efremov, K. Goeke and P. Schweitzer, Phys. Lett. B 522, 37 (2001) arXiv:hep-ph/0108213 and 544, 389E (2002).

[71] A. V. Efremov, K. Goeke and P. Schweitzer, Eur. Phys. J. C 24 (2002) 407 arXiv:hep-ph/0112166]; Phys. Lett. B 568 (2003) 63 arXiv:hep-ph/0303062; Phys. Rev. D 67, 114014 (2003) arXiv:hep-ph/0208124.

[72] B. Q. Ma, I. Schmidt and J. J. Yang, Phys. Rev. D 66, 094001 (2002) arXiv:hep-ph/0209114.

[73] A. Bacchetta, P. J. Mulders and F. Pijlman, Phys. Lett. B 595 (2004) 309.

[74] F. Yuan, Phys. Lett. B 589 (2004) 28.

[75] L. P. Gamberg, D. S. Hwang and K. A. Oganessyan, Phys. Lett. B 584 (2004) 276.

[76] A. Metz and M. Schlegel, Eur. Phys. J. A 22 (2004) 489, Annalen Phys. 13 (2004) 699.

[77] A. V. Afanasev and C. E. Carlson, Phys. Rev. D 74, 114027 (2006) arXiv:hep-ph/0603269.

[78] L. P. Gamberg, D. S. Hwang, A. Metz and M. Schlegel, Phys. Lett. B 639, 508 (2006) arXiv:hep-ph/0604022.

[79] R. Jakob, P. J. Mulders and J. Rodrigues, Nucl. Phys. A 626, 937 (1997) arXiv:hep-ph/9704335; arXiv:hep-ph/9707340

[80] H. Avakian, A. V. Efremov, P. Schweitzer and F. Yuan, Phys. Rev. D 78, 114024 (2008) arXiv:0805.3355 [hep-ph]].

[81] B. Pasquini, S. Cazzaniga and S. Boffi, Phys. Rev. D 78, 034025 (2008) arXiv:0806.2298 [hep-ph]].

[82] A. V. Efremov, P. Schweitzer, O. V. Teryaev and P. Zavada, Phys. Rev. D 80, 014021 (2009) [arXiv:0903.3490 [hep-ph]], AIP Conf. Proc. 1149, 547 (2009) arXiv:0812.3246 [hep-ph]].

[83] A. V. Efremov, P. Schweitzer, O. V. Teryaev and P. Zavada, arXiv:0912.3380 [hep-ph].

[84] J. She, J. Zhu and B. Q. Ma, Phys. Rev. D 79 (2009) 054008.

[85] A. Bacchetta, F. Conti and M. Radici, arXiv:0807.0323 [hep-ph].

[86] S. Boffi, A. V. Efremov, B. Pasquini and P. Schweitzer, Phys. Rev. D 79, 094012 (2009) arXiv:0903.1271 [hep-ph]], AIP Conf. Proc. 1149, 471 (2009) arXiv:0903.1830 [hep-ph]], Mod. Phys. Lett. A 24, 2903 (2009) arXiv:0910.1677[hep-ph]], arXiv:0912.1761 [hep-ph].

[87] H. Avakian, A. V. Efremov, P. Schweitzer, O. V. Teryaev, F. Yuan and P. Zavada, Mod. Phys. Lett. A 24, 2995 (2009) arXiv:0910.3181 [hep-ph]].

[88] M. Wakamatsu, Phys. Rev. D 79, 094028 (2009) arXiv:0903.1886 [hep-ph]].

[89] S. Meissner, A. Metz and K. Goeke, Phys. Rev. D 76, 034002 (2007) arXiv:hep-ph/0703176.

[90] L. P. Gamberg, G. R. Goldstein and M. Schlegel, Phys. Rev. D 77 (2008) 094016.

[91] F. Yuan, Phys. Lett. B 575, 45 (2003) arXiv:hep-ph/0308157.

[92] A. Courtoy, S. Scopetta and V. Vento, Phys. Rev. D 79, 074001 (2009) arXiv:0811.1191 [hep-ph]].

A. Courtoy, F. Fratini, S. Scopetta and V. Vento, Phys. Rev. D 78, 034002 (2008) arXiv:0801.4347 [hep-ph]].

[93] I. O. Cherednikov, U. D’Alesio, N. I. Kochelev and F. Murgia, Phys. Lett. B 642, 39 (2006) arXiv:hep-ph/0606238.

[94] L. P. Gamberg, G. R. Goldstein and M. Schlegel, arXiv:0708.2580 [hep-ph] and arXiv:0708.0324 [hep-ph].

[95] R. Kundu and A. Metz, Phys. Rev. D 65 (2002) 014009. M. Schlegel and A. Metz, arXiv:hep-ph/0406289

[96] K. Goeke, A. Metz, P. V. Pobylitsa, M. V. Polyakov, Phys. Lett. B 567 (2003) 27.

[97] A. Kotzinian, B. Parsamyan and A. Prokudin, Phys. Rev. D 73, 114017 (2006) arXiv:hep-ph/0603194.

[98] H. Avakian, A. V. Efremov, K. Goeke, A. Metz, P. Schweitzer and T. Teckentrup, Phys. Rev. D 77, 014023 (2008) arXiv:0709.3253 [hep-ph]].

[99] A. Metz, P. Schweitzer and T. Teckentrup, Phys. Lett. B 680, 141 (2009) arXiv:0810.5212 [hep-ph]].

[100] T. Teckentrup, A. Metz and P. Schweitzer, Mod. Phys. Lett. A 24, 2950 (2009) arXiv:0910.2567 [hep-ph]].

[101] A. Accardi, A. Bacchetta, W. Melnitchouk and M. Schlegel, JHEP 0911, 093 (2009) arXiv:0907.2942 [hep-ph]].

A. Accardi, A. Bacchetta and M. Schlegel, AIP Conf. Proc. 1155, 35 (2009) arXiv:0905.3118 [hep-ph]].

[102] J. P. Ralston and D. E. Soper, Nucl. Phys. B 152 (1979) 109.

[103] R. L. Jaffe and X. D. Ji, Nucl. Phys. B 375 (1992) 527, and Phys. Rev. Lett. 67, 552 (1991).

[104] A. Chodos, R. L. Jaffe, K. Johnson, C. B. Thorn and V. F. Weisskopf, Phys. Rev. D 9, 3471 (1974).

[105] R. L. Jaffe, Phys. Rev. D 11, 1953 (1975).

[106] L. S. Celenza and C. M. Shakin, Phys. Rev. C 27, 1561 (1983) [Erratum-ibid. C 39, 2477 (1989)].

[107] G. Karl and J. E. Paton, Phys. Rev. D 30, 238 (1984).

[108] A. W. Schreiber, A. I. Signal and A. W. Thomas, Phys. Rev. D 44, 2653 (1991).

[109] S. Wandzura and F. Wilczek, Phys. Lett. B 72 (1977) 195.

[110] X. Zheng et al. [Jefferson Lab Hall A Collaboration], Phys. Rev. C 70, 065207 (2004) arXiv:nucl-ex/0405006. M. Amarian et al. [Jefferson Lab E94-010 Collaboration], Phys. Rev. Lett. 92 (2004) 022301 arXiv:hep-ex/0310003]. P. L. Anthony et al. [E155 Collaboration], Phys. Lett. B 553 (2003) 18 arXiv:hep-ex/0204028.

[111] J. Balla, M. V. Polyakov and C. Weiss, Nucl. Phys. B 510, 327 (1998) arXiv:hep-ph/9707515.

B. Dressler and M. V. Polyakov, Phys. Rev. D 61, 097501 (2000) arXiv:hep-ph/9912376.

[112] M. Gockeler et al., Phys. Rev. D 63, 074506 (2001), Phys. Rev. D 72, 054507 (2005) arXiv:hep-lat/0506017.

[113] I. V. Anikin and O. V. Teryaev, Phys. Lett. B 509, 95 (2001) arXiv:hep-ph/0102209. 
A. V. Radyushkin and C. Weiss, Phys. Rev. D 63, 114012 (2001) arXiv:hep-ph/0010296.

N. Kivel, M. V. Polyakov, A. Schäfer and O. V. Teryaev, Phys. Lett. B 497, 73 (2001) arXiv:hep-ph/0007315].

J. Blümlein and A. Tkabladze, Nucl. Phys. B 553 (1999) 427 arXiv:hep-ph/9812478.

J. Blümlein and N. Kochelev, Nucl. Phys. B 498 (1997) 285 arXiv:hep-ph/9612318.

O. V. Teryaev, in the Proceedings of "Prospects of Spin Physics at HERA, Zeuthen, Germany, 28-31 Aug 1995,"

Eds. J. Blümlein and W.-D. Nowak, Hamburg, Germany, 1995 (DESY-95-200), pp. 132-142 arXiv:hep-ph/0102296.

P. Ball and V. M. Braun, Phys. Rev. D 54, 2182 (1996) arXiv:hep-ph/9602323.

[114] A. V. Efremov and P. Schweitzer, JHEP 0308 (2003) 006 [arXiv:hep-ph/0212044].

[115] A. I. Signal, Nucl. Phys. B 497 (1997) 415 arXiv:hep-ph/9610480.

[116] V. Barone, A. Drago and P. G. Ratcliffe, Phys. Rept. 359 (2002) 1 arXiv:hep-ph/0104283.

[117] B. Pasquini, M. Pincetti and S. Boffi, Phys. Rev. D 72, 094029 (2005) arXiv:hep-ph/0510376]; Phys. Rev. D 76, 034020 (2007) arXiv:hep-ph/0612094.

[118] M. Wakamatsu, Phys. Lett. B 487, 118 (2000), Phys. Lett. B 509, 59 (2001) arXiv:hep-ph/0012331.

[119] P. Schweitzer, Phys. Rev. D 67, 114010 (2003) arXiv:hep-ph/0303011.

M. Wakamatsu and Y. Ohnishi, Phys. Rev. D 67, 114011 (2003), Phys. Rev. D 69, 114002 (2004) arXiv:hep-ph/0312044. C. Cebulla, J. Ossmann, P. Schweitzer and D. Urbano, Acta Phys. Polon. B 39, 609 (2008) arXiv:0710.3103 [hep-ph]].

[120] M. Burkardt and Y. Koike, Nucl. Phys. B 632, 311 (2002) arXiv:hep-ph/0111343.

[121] A. Mukherjee, arXiv:0912.1446 [hep-ph].

[122] J. D. Jackson, G. G. Ross and R. G. Roberts, Phys. Lett. B 226, 159 (1989).

[123] P. Zavada, Phys. Rev. D 55, 4290 (1997) arXiv:hep-ph/9609372; Phys. Rev. D 65, 054040 (2002) arXiv:hep-ph/0106215. Phys. Rev. D 67, 014019 (2003) arXiv:hep-ph/0210141], Eur. Phys. J. C 52, 121 (2007) [arXiv:0706.2988 [hep-ph]].

[124] A. V. Belitsky, proceedings of "31st PNPI Winter School on Nuclear and Particle Physics, St. Petersburg, Russia, 1997", Ed. V. A. Gordeev, pp. 369-455 arXiv:hep-ph/9703432.

[125] X. D. Ji, W. Melnitchouk and X. Song, Phys. Rev. D 56, 5511 (1997) arXiv:hep-ph/9702379.

[126] P. Schweitzer, D. Urbano, M. V. Polyakov, C. Weiss, P. V. Pobylitsa and K. Goeke, Phys. Rev. D 64 (2001) 034013 arXiv:hep-ph/0101300. K. Goeke et al., Acta Phys. Polon. B 32, 1201 (2001) arXiv:hep-ph/0001272.

M. Wakamatsu and T. Kubota, Phys. Rev. D 60, 034020 (1999) arXiv:hep-ph/9809443.

[127] A. W. Thomas, Phys. Rev. Lett. 101, 102003 (2008) arXiv:0803.2775 [hep-ph]].

[128] M. G. Alekseev et al. [COMPASS Collaboration], arXiv:1001.4654 [hep-ex].

A. Airapetian et al. [HERMES Collaboration], Phys. Rev. D 75, 012007 (2007) arXiv:hep-ex/0609039.

B. Adeva et al. [Spin Muon Collaboration], Phys. Rev. D 58, 112001 (1998).

[129] R. S. Pasechnik, D. V. Shirkov and O. V. Teryaev, Phys. Rev. D 78, 071902 (2008) arXiv:0808.0066 [hep-ph]].

[130] Ph. Hägler, Physics Reports (2010), in press arXiv:0912.5483 [hep-lat]].

[131] M. Wakamatsu and Y. Nakakoji, Phys. Rev. D 77, 074011 (2008) arXiv:0712.2079 [hep-ph]].

M. Wakamatsu, arXiv:0908.0972 [hep-ph].

[132] M. Stratmann, Z. Phys. C 60, 763 (1993).

[133] B. Q. Ma and I. Schmidt, Phys. Rev. D 58, 096008 (1998) arXiv:hep-ph/9808202.

B. Q. Ma, I. Schmidt and J. Soffer, Phys. Lett. B 441, 461 (1998) arXiv:hep-ph/9710247.

[134] F. Landry, R. Brock, P. M. Nadolsky and C. P. Yuan, Phys. Rev. D 67 (2003) 073016 arXiv:hep-ph/0212159. 